\title{
Definition of an invariant Lamb-vector-based aerodynamic force breakdown using far-field flow symmetries
}

\author{
Camille Fournis* and Didier Bailly ${ }^{\dagger}$ \\ ONERA-The French Aerospace Lab, 92190 Meudon, France \\ Renato Tognaccini ${ }^{\ddagger}$ \\ Università di Napoli Federico II, Napoli, 80125, Italia
}

\begin{abstract}
Some recent developments in the Lamb-vector-based aerodynamic force breakdown used the concept of vortex force in order to define the lift and to decompose the drag into lift-induced drag and profile drag. However, the Lamb vector formulation involves moment transformations and the associated force breakdown may depend on the reference point adopted for their computation. Yet, the force acting on an airplane cannot be dependent on this point. Thus, a systematic method based on the far-field flow symmetries is here proposed in order to eliminate this dependence. Those symmetries also allow for a better understanding of the progressive invariance of the drag decomposition with respect to the size of the integration domain. Finally, a reference-point-invariant formulation is defined and tested for a two-dimensional transonic flow around an OAT15A airfoil and a three-dimensional flow around the NASA Common Research Model (CRM) under cruise flight conditions.
\end{abstract}

\section{Nomenclature}

$\begin{array}{ll}a & =\text { acceleration of a fluid particle } \\ c & =\text { chord } \\ c_{r e f} & =\text { reference chord } \\ C_{D} & =\text { drag coefficient } \\ C_{D_{i}} & =\text { lift-induced drag coefficient } \\ C_{D_{v}} & =\text { viscous drag coefficient } \\ C_{D_{w}} & =\text { wave drag coefficient } \\ C_{L} & =\text { lift coefficient } \\ d & =\text { distance from the body surface } S_{b}\end{array}$

Presented as paper 2020-1996 at the AIAA 2020 SciTech Forum, Orlando, FL, 6-10 January 2020.

*Ph.D student, Aerodynamics, Aeroelasticity and Acoustics Department, camille.fournis@onera.fr.

${ }^{\dagger}$ Research Engineer, Aerodynamics, Aeroelasticity and Acoustics Department, didier.bailly@onera.fr.

$¥$ Associate Professor, Dipartimento di Ingegneria Industriale, renato.tognaccini@unina.it, AIAA Senior Member. 


$$
\begin{aligned}
& D_{i} \quad=\text { lift-induced drag } \\
& D_{P} \quad=\text { profile drag } \\
& \left(\boldsymbol{e}_{X}, \boldsymbol{e}_{Y}, \boldsymbol{e}_{Z}\right)=\text { body-fixed frame } \\
& \left(\boldsymbol{e}_{x}, \boldsymbol{e}_{y}, \boldsymbol{e}_{z}\right)=\text { wind-fixed frame } \\
& \boldsymbol{F} \quad=\text { aerodynamic force } \\
& H \quad=\text { total enthalpy } \\
& L \quad=\text { lift } \\
& \boldsymbol{l}=\text { lamb vector } \boldsymbol{l}=\boldsymbol{\omega} \times \boldsymbol{u} \\
& M_{\infty} \quad=\quad \text { freestream Mach number } \\
& \boldsymbol{m}_{\rho} \quad=\text { compressibility correction term } \boldsymbol{m}_{\rho}=\frac{r}{n-1} \times\left(\nabla \rho \times \nabla\left(\frac{u^{2}}{2}\right)\right) \\
& n=\text { unit normal } \\
& n=\text { dimension of the physical space } n=2,3 \\
& p \quad=\text { pressure } \\
& p_{\infty} \quad=\text { freestream pressure } \\
& R \quad=\text { perfect gas constant } \\
& r \quad=\text { position vector } \\
& \text { Re } \quad=\text { Reynolds number } \\
& s \quad=\text { entropy } \\
& S_{b} \quad=\text { body surface } \\
& S_{e} \quad=\text { external boundary } \\
& S_{e}^{*} \quad=\text { external boundary of the fluid domain extension } \\
& S_{w} \quad=\text { portion of } S_{e} \text { crossing the wake } \\
& \boldsymbol{u}=\text { velocity vector } \boldsymbol{u}=u_{x} \boldsymbol{e}_{x}+u_{y} \boldsymbol{e}_{y}+u_{z} \boldsymbol{e}_{z} \\
& u^{2}=\text { velocity squared } u^{2}=u_{x}^{2}+u_{y}^{2}+u_{z}^{2} \\
& \boldsymbol{U}_{\infty} \quad=\text { freestream velocity } \boldsymbol{U}_{\infty}=U_{\infty} \boldsymbol{e}_{x} \\
& (X, Y, Z) \quad=\text { body-fixed coordinate system } \\
& (x, y, z) \quad=\text { wind-fixed coordinate system } \\
& \alpha \quad=\text { angle of attack } \\
& \Delta H=\text { total enthalpy variation with respect to its freestream value } \\
& \Delta s \quad=\text { entropy variation with respect to its freestream value } \\
& \Delta \Omega \quad=\text { fluid domain extension } \\
& \Delta \Omega_{w} \quad=\text { part of } \Delta \Omega \text { crossing the wake }
\end{aligned}
$$




$$
\begin{array}{ll}
\mu & =\text { dynamic viscosity } \\
\Omega & =\text { fluid domain } \\
\omega & =\text { vorticity vector } \omega=\nabla \times \boldsymbol{u} \\
\rho & =\text { density } \\
\rho_{\infty} & =\text { freestream density } \\
\tau & =\text { viscous stress tensor } \\
\nabla & =\text { gradient operator } \nabla=\frac{\partial}{\partial x} \boldsymbol{e}_{x}+\frac{\partial}{\partial y} \boldsymbol{e}_{y}+\frac{\partial}{\partial z} \boldsymbol{e}_{z}
\end{array}
$$

\section{Introduction}

HE determination of the total aerodynamic force acting on a body has been of paramount importance since the birth of aviation in the late nineteenth and early twentieth century. An accurate computation of the aerodynamic drag is a major issue when designing an aircraft since it has a significant impact on its global performance, total weight and fuel consumption. Therefore this topic has been a matter of research for decades in order to reduce the drag generated by commercial airplanes. The development of various methods to compute the aerodynamic force acting on an aircraft allowed for a better physical understanding of the drag and enabled the engineers to improve their design.

There are two ways of computing the aerodynamic force: the near-field and the far-field approach. The former consists in integrating the pressure and shear stresses acting on the body skin $S_{b}$ whereas the latter is based on the momentum balance in a control volume of fluid $\Omega$ and its external boundary $S_{e}$ (see Fig 1 :

$$
\boldsymbol{F}=-\oint_{S_{b}}(-p \mathbf{1}+\boldsymbol{\tau}) \cdot \boldsymbol{n} \mathrm{d} S=-\int_{\Omega} \rho \boldsymbol{a} \mathrm{d} v+\oint_{S_{e}}(-p \mathbf{1}+\tau) \cdot \boldsymbol{n} \mathrm{d} S
$$

where $\boldsymbol{a}$ is the acceleration of a fluid particle, $\boldsymbol{\tau}$ is the viscous stress tensor and $\boldsymbol{n}$ is the unit normal to the surface, pointing outside the fluid. The near-field approach only provides a mechanical breakdown into friction drag and pressure drag.

The far-field approach provides several advantages:

- A decomposition of the aerodynamic drag into several terms related to the physical phenomena occuring in the flow: trailing vortices, viscous wakes and shock waves. Hence the total drag is given by the sum of three contributions, namely the induced drag, the viscous drag and the wave drag: $C_{D}=C_{D_{i}}+C_{D_{v}}+C_{D_{w}}$.

- The identification of the local flow structures where the different drag contributions are generated in the flow field.

- An estimate of the spurious drag which allows for a more accurate calculation of the total drag when dealing with numerical computations.

The first far-field formula known is the celebrated Joukowski's formula [1] also contained in Kutta's unpublished 


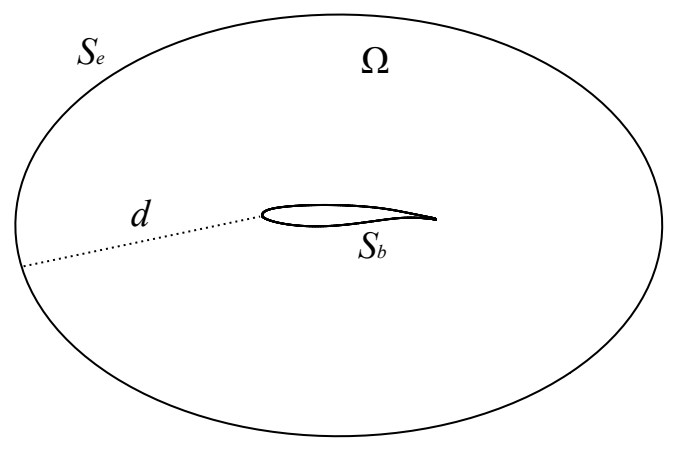

(a) $S_{e}$ defined by the distance from the body

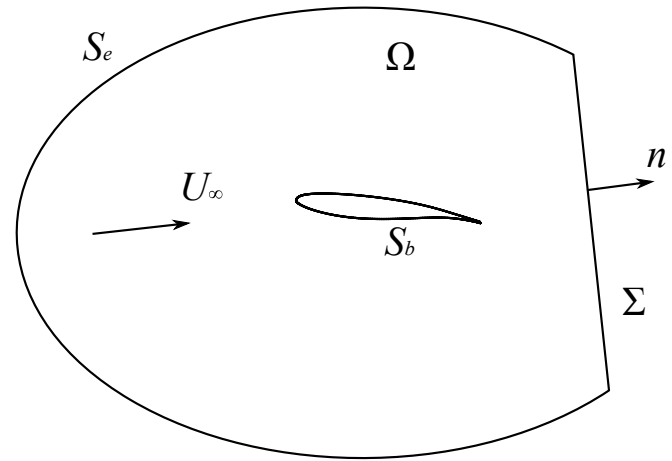

(b) $S_{e}$ defined with a wake plane $\Sigma$

Fig. 1 Fluid domain and its boundaries

thesis dissertation of 1902 [2] and now referred to as Kutta-Joukowski theorem. This formula relates the lift to the circulation generated by an airfoil in the flow but cannot provide the drag: this is the famous d'Alembert's paradox which partly explains why this theory was at first left on the sidelines. Indeed, this theory was developed for two-dimensional inviscid flows for which there should be no circulation nor vorticity creation according to Kelvin's theorem [3]. The circulation is actually set by the Kutta condition which accounts for the viscous effects occuring in the boundary layer and states that the flow must leave the airfoil at the trailing edge. The Kutta-Joukowski lift theorem was later generalized to the study of lifting wings by Prandtl [4] in which the addition of a velocity perturbation allowed him to define a drag contribution due to the presence of vortices: the lift-induced drag. At the same period, Filon's works [5] led to a drag formula valid in the case of viscous incompressible flows. Very recently, Liu et al. [6, 7] extended the use of the Kutta-Joukowski lift theorem and Filon's drag formula to compressible steady viscous flows and derived a unified force formula along with its testable version. Schmitz [8] investigated how the Kutta-Joukowski formula is altered in viscous flows and derived a finite-domain correction term based on the perturbation Lamb vector which accounts for viscous effects in the wake.

The use of experimental fluid dynamics in aircraft design emphasized the need for formulae that could provide the lift-induced drag and the profile drag from wake surveys (a thorough review can be found in [9]). In his inviscid lifting line theory, Prandtl assumed the wake vorticity to be confined in a vortex sheet of infinitesimal width with no rolling-up. Later on, Maskell [10] proposed a definition of the lift-induced drag valid in the case of incompressible flows with a non planar vortex sheet [11] which is still widely used today in wind-tunnel experiments and CFD computations drag breakdown postprocessing tools [12--18]. Betz [19] and Taylor [20] had previously linked the profile drag to the total pressure loss in incompressible flows. Jones [21] assumed more restrictive hypotheses in order to derive a profile drag formula also applicable to compressible flows still in use in planar wake surveys.

The development of drag breakdown formulations able to address three-dimensional compressible flows then became of paramount importance in order to enhance the robustness and accuracy of drag predictions. In 1956, 
Oswatitsch [22] derived a profile drag formula based on the entropy creation within boundary layers and across shock waves. This concept of entropy drag was later generalized with the development of thermodynamic formulae which can provide a consistent breakdown of the profile drag into viscous and wave drag contributions [23-26] (a detailed review can be found in [27]). The main drawback of those formulations lies in the fact that they do not define explicitly and cannot accurately compute the lift-induced drag since it is found by subtracting the profile drag to the total drag. Therefore, how to define the lift-induced drag in compressible viscous flows still remained an open issue as pointed out by Spalart [28]. Another approach based on partial pressure fields was later developed by Schmitz and Coder [29] for incompressible flows before it was extended to the compressible regime by Schmitz [30].

The vorticity-based formulations form another family of the far-field methods and have been subject to intense research in the very recent years (an exhaustive summary is presented in [11]) since they provide a consistent definition of the lift and the lift-induced drag. The vortex force theory was first introduced by Prandtl [4] and was discussed by Saffman [31] in the case of an incompressible steady inviscid flow. In their derivations the pressure is replaced by the kinetic energy upon using the Bernoulli equation and the aerodynamic force is expressed with the Lamb vector field $l=\omega \times \boldsymbol{u}$ where $\omega=\nabla \times \boldsymbol{u}$ is the vorticity. The extension to the relevant case of the body wake convected infinitely downstream also including the steady case was conducted by Wu et al. [32, 33] where the pressure field is eliminated by using the derivative moment transformation Eq. A.2 (defined in Appendix A):

$$
\boldsymbol{F}=-\int_{\Omega} \rho \boldsymbol{l} \mathrm{d} v-\frac{1}{n-1} \oint_{S_{e}} \boldsymbol{r} \times(\boldsymbol{n} \times \rho \boldsymbol{l}) \mathrm{d} S+\frac{1}{n-1} \oint_{S_{e}} \boldsymbol{r} \times(\boldsymbol{n} \times \nabla \cdot \boldsymbol{\tau}) \mathrm{d} S+\oint_{S_{e}} \boldsymbol{\tau} \cdot \boldsymbol{n} \mathrm{d} S
$$

where $n=2,3$ is the dimension of the space and $\boldsymbol{r}$ is the position vector. A compressible version of the above-written formulation was suggested by Wu et al. [32] (p. 621) and by Liu et al. [34] who defined a compressible Lamb vector $\rho \boldsymbol{l}-\frac{u^{2}}{2} \nabla \rho$ such that:

$$
\boldsymbol{F}=-\int_{\Omega}\left(\rho \boldsymbol{l}-\frac{u^{2}}{2} \nabla \rho\right) \mathrm{d} v-\frac{1}{n-1} \oint_{S_{e}} \boldsymbol{r} \times\left[\boldsymbol{n} \times\left(\rho \boldsymbol{l}-\frac{u^{2}}{2} \nabla \rho\right)\right] \mathrm{d} S+\frac{1}{n-1} \oint_{S_{e}} \boldsymbol{r} \times(\boldsymbol{n} \times \nabla \cdot \boldsymbol{\tau}) \mathrm{d} S+\oint_{S_{e}} \boldsymbol{\tau} \cdot \boldsymbol{n} \mathrm{d} S
$$

In incompressible flows, the integration can be limited to regions where the vorticity is nonzero since only the Lamb vector appears whereas in compressible flows, one loses the compactness of the integrands because of the term $\frac{u^{2}}{2} \nabla \rho$. Hence, Mele and Tognaccini [35] used Eq. [A.1] (see Appendix A) in order to express Eq. (3) as follows:

$$
\boldsymbol{F}=\boldsymbol{F}_{\rho l}+\boldsymbol{F}_{m_{\rho}}+\boldsymbol{F}_{S_{e}}+\boldsymbol{F}_{\tau}
$$


where

$$
\begin{aligned}
\boldsymbol{F}_{\rho l} & =-\int_{\Omega} \rho \boldsymbol{l} \mathrm{d} v \\
\boldsymbol{F}_{m_{\rho}} & =-\frac{1}{n-1} \int_{\Omega} \boldsymbol{r} \times\left(\nabla \rho \times \nabla\left(\frac{u^{2}}{2}\right)\right) \mathrm{d} v=-\int_{\Omega} \boldsymbol{m}_{\rho} \mathrm{d} v \\
\boldsymbol{F}_{S e} & =-\frac{1}{n-1} \oint_{S_{e}} \boldsymbol{r} \times(\boldsymbol{n} \times \rho \boldsymbol{l}) \mathrm{d} S \\
\boldsymbol{F}_{\tau} & =\frac{1}{n-1} \oint_{S_{e}} \boldsymbol{r} \times(\boldsymbol{n} \times \nabla \cdot \boldsymbol{\tau}) \mathrm{d} S+\oint_{S_{e}} \boldsymbol{\tau} \cdot \boldsymbol{n} \mathrm{d} S
\end{aligned}
$$

$\boldsymbol{F}_{\rho l}$ is the vortex force which provides the lift $L$ and the lift-induced drag $D_{i}$ in steady incompressible flows [36], $\boldsymbol{F}_{m_{\rho}}$ is a compressibility correction term to the vortex force which contributes to $L$ and $D_{i}$ in steady compressible flows [35] and $\boldsymbol{F}_{S e}$ provides the profile drag $D_{P}$ as noticed by several authors [32, 35]. Finally, for a large enough control volume, $S_{e}$ retreats to flow regions where viscous stresses become negligible such that the contribution of $\boldsymbol{F}_{\tau}$ can be dropped [36, 37].

The profile drag consists of a viscous and a wave contribution: the viscous drag is produced in the boundary layers while the wave drag is produced across shock waves. Therefore, distinguishing the two contributions turns out to be tough as noticed by Mele et al. [38] who proposed to define the wave drag as the surface integral $\boldsymbol{F}_{S e}$ on a wake plane directly downstream of the shock. The viscous drag was then computed subtracting the wave drag to the profile drag. Later on, Ostieri and Tognaccini [39] pointed out that this definition tends to systematically overestimate the wave drag. Hence, they used Gauss theorem in order to transform the surface integral Eq. (7) into a volume integral limited to the boundary layer and shock wave regions $\Omega_{v}$ and $\Omega_{s w}$. It was surmised that the volume $\Omega_{s w}$ should contain the Lamb vector field produced in the near wake of the shock in order to correctly compute the wave drag [39].

Very recently, Zou et al. [40] investigated the dependence of the lift-induced drag to the choice of the integration domain and proposed a new formula which is less sensitive and improves the robustness of the Lamb vector formulation. Similarly, Kang et al. [41] studied the sensitivity of the Lamb-vector-based drag predictions in viscous and inviscid flows along with the effect of the Reynolds number.

Hence, the Lamb-vector-based decomposition is very promising since it relies on an exact formula and might well predict all the contributions of the drag along with the lift. However, there are still flaws to tackle in order to enhance its robustness:

- The dependence on the reference point adopted for the computation of moment transformations.

- The sensitivity to the integration domain.

- A robust wave drag definition.

In this paper, the focus is given to the first two points. Section III sets the equations and presents numerical results which testify that the decomposition Eq.(4) is sensitive to the reference point and to the size of the control volume. 
Section IV investigates the natural far-field symmetries satisfied by the flow inside (IV.A) and outside the wake (IV.B). In the context of those symmetries, subsection V.A shows how the decomposition progressively becomes invariant to the choice of the reference point (V.A.1) and to the size of the control volume (V.A.2) in the far field. Finally subsections V.B and V.C establish and test a novel formulation which is reference-point-invariant in the whole flow domain.

\section{The limitations of the present formulation}

\section{A. The dependence on the reference point}

\section{Presentation of the problem}

The surface integral $\boldsymbol{F}_{S_{e}}$ can be limited to the wake where the Lamb vector $\boldsymbol{l}$ is nonzero: in practice, the aforementioned authors adopted a wake plane perpendicular to the freestream velocity $\boldsymbol{U}_{\infty}=U_{\infty} \boldsymbol{e}_{x}$ (Trefftz plane) for the computation of the profile drag. To do so, one must first proceed to an interpolation of the numerical solution onto this plane and may lose accuracy. Furthermore, the use of derivative moment transformations Eq.A.1] and Eq. A.2) introduce the position vector $\boldsymbol{r}$ in Eq. (6) and Eq. (7). The total force $\boldsymbol{F}$ given by Eq. (4) is independent of the reference point [32] but the terms of the decomposition $\boldsymbol{F}_{m_{\rho}}, \boldsymbol{F}_{S_{e}}$ and $\boldsymbol{F}_{\tau}$ individually depend on its choice which raises the question: is the force decomposition dependent on the reference point adopted for the computation of moment transformations? In other words, is the breakdown likely to be changed if one chooses a reference point different from the origin of the frame?

One way to circumvent this issue has hitherto consisted in setting this point on the wake plane adopted for profile drag computation [35, 38, 41, 42], which also reduces $\boldsymbol{F}_{S_{e}}$ to a drag contribution only. However, when $\boldsymbol{r}$ is shifted by a constant $\boldsymbol{r}_{0}$ (i.e. $\boldsymbol{r} \rightarrow \boldsymbol{r}+\boldsymbol{r}_{0}$ ) in $\boldsymbol{F}_{S_{e}}$ and $\boldsymbol{F}_{m_{\rho}}$, an additional term is generated:

$$
\begin{aligned}
\boldsymbol{F}_{S_{e}} & \rightarrow \boldsymbol{F}_{S_{e}}-\frac{\boldsymbol{r}_{0}}{n-1} \times \oint_{S_{e}} \boldsymbol{n} \times \rho \boldsymbol{l} \mathrm{d} S \\
\boldsymbol{F}_{m_{\rho}} & \rightarrow \boldsymbol{F}_{m_{\rho}}+\frac{\boldsymbol{r}_{0}}{n-1} \times \oint_{S_{e}} \boldsymbol{n} \times \frac{u^{2}}{2} \nabla \rho \mathrm{d} S \\
& =\boldsymbol{F}_{m_{\rho}}+\frac{\boldsymbol{r}_{0}}{n-1} \times \int_{\Omega} \nabla\left(\frac{u^{2}}{2}\right) \times \nabla \rho \mathrm{d} v
\end{aligned}
$$

Although the additional terms cancel out in the total force computation, the expression of each individual term is changed which makes it tough to correctly define the different force contributors:

$$
\begin{aligned}
\boldsymbol{F} & =\boldsymbol{F}_{\rho l}+\boldsymbol{F}_{m_{\rho}}+\frac{\boldsymbol{r}_{0}}{n-1} \times \oint_{S_{e}} \boldsymbol{n} \times \frac{u^{2}}{2} \nabla \rho \mathrm{d} S \\
& +\boldsymbol{F}_{S_{e}}-\frac{\boldsymbol{r}_{0}}{n-1} \times \oint_{S_{e}} \boldsymbol{n} \times \rho \boldsymbol{l} \mathrm{d} S
\end{aligned}
$$




\section{Computation of $C_{D_{S_{e}}}$ for various positions of the reference point}

To illustrate the problem, a numerical computation was performed using RANS equations, Spalart-Allmaras turbulence model and Jameson's scheme (cell-centered, $2^{\text {nd }}$ and $4^{\text {th }}$ order artificial viscosities) in the AIRBUSSAFRAN-ONERA elsA solver [43] for a transonic steady viscous flow around an OAT15A airfoil (see Fig 2) with a Mach number $M_{\infty}=0.724$, a Reynolds number $R e=3 \times 10^{6}$ and an incidence $\alpha=1.15^{\circ}\left(C_{L} \approx 0.73\right)$. The mesh has 312,082 nodes and the far field lies 50,000 airfoil chords away $(c=1 \mathrm{~m})$ from the body surface. The computation was converged to a drop in residuals of $10^{13}$. The postprocess of the CFD solution evidenced $y+\approx 0.5$ for the first layer of grid cells around the airfoil. A grid convergence study on the OAT15A airfoil is presented in Appendix C. The numerical solution was then postprocessed with a FORTRAN code in order to compute the aerodynamic force acting on the airfoil. The external boundary $S_{e}$ is defined by the distance $d$ between the nodes where integration is performed and the body surface $S_{b}$ as sketched in Fig $2 \mathrm{~b} \quad \boldsymbol{F}_{S_{e}}$ and $\boldsymbol{F}_{m_{\rho}}$ were computed with Eqs. (6) and (7) and the drag and lift coefficients are defined as follows:

$$
\begin{aligned}
C_{D_{\bullet}} & =\frac{2 D_{\bullet}}{\rho_{\infty} U_{\infty} S_{\text {ref }}} \\
C_{L_{\bullet}} & =\frac{2 L_{\bullet}}{\rho_{\infty} U_{\infty} S_{\text {ref }}}
\end{aligned}
$$

where $S_{r e f}$ is a reference area. Finally, the reference point will be shifted from the origin of the frame by 0, 25 and 50 chord lengths in the $X$ and $Z$ directions.

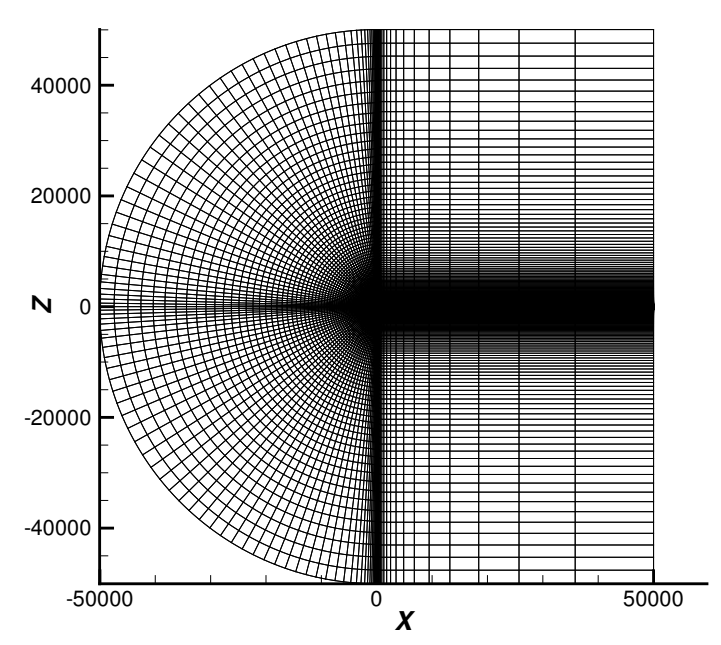

(a) H-C type mesh with two blocks (1 point over 4)

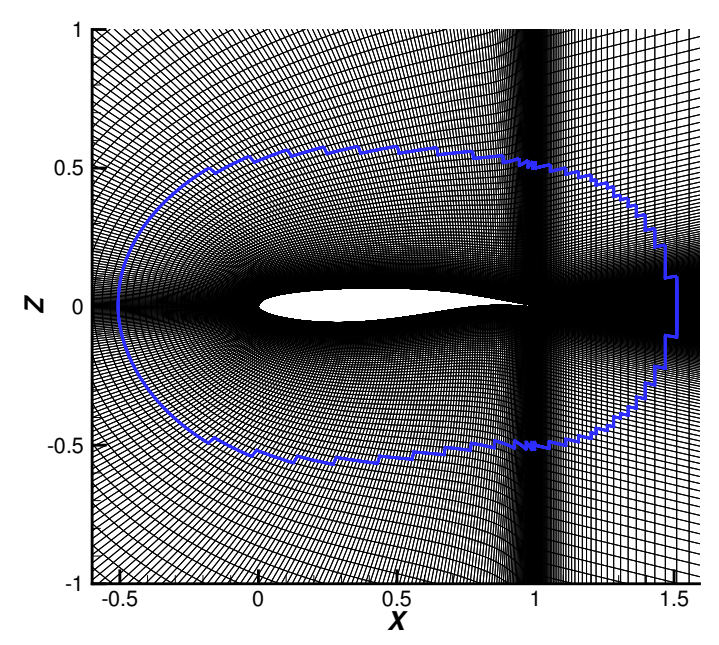

(b) Near-wall mesh resolution $\left(S_{e}\right.$ in blue)

Fig. 2 OAT15A airfoil grid

The evolution of $C_{D_{S}}$ with respect to the distance $d$ for various $\left(X_{0}, Z_{0}\right)$-locations of the reference point is plotted 
in Fig 3 It is clear that changing the reference point leads to significant disparities in the assessment of the drag when $S_{e}$ is placed close to the airfoil. Nevertheless, those figures also highlight that these disparities vanish as $S_{e}$ extends further away from the body.

Therefore it is of high interest to identify the mechanisms that lead to a reference-point-invariant aerodynamic force breakdown. According to Eq.99, the behavior exhibited in Fig 3 suggests that for a sufficiently large $S_{e}$ :

$$
\oint_{S_{e}} \boldsymbol{n} \times \rho \boldsymbol{l} \mathrm{d} S \approx \mathbf{0}
$$

This condition is actually fulfilled in the far wake since the wake spreads out because of viscous diffusion and progressively satisfies symmetry properties with respect to the $(x, y)$-plane. Indeed, Fournis et al. [44] considered a simplified two-dimensional example to illustrate the symmetries gradually verified by the Lamb vector $\rho \boldsymbol{l}$ and the term $\frac{u^{2}}{2} \nabla \rho$ in the far wake.

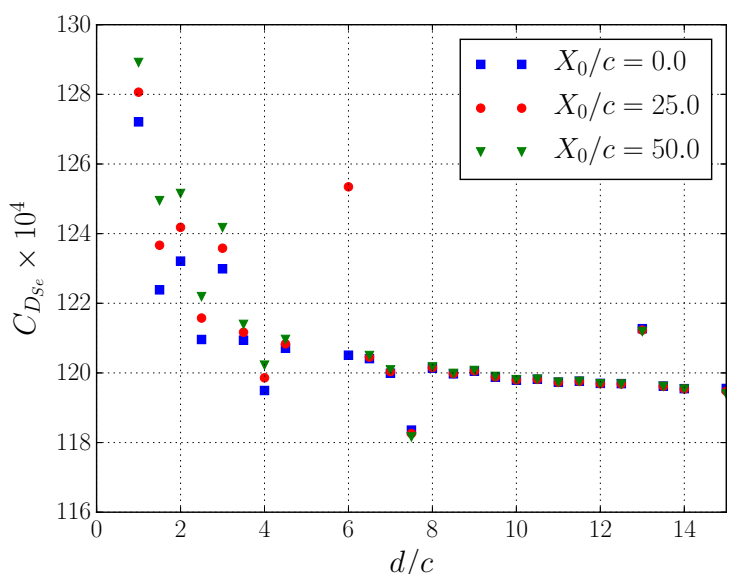

(a) $X \rightarrow X+X_{0}$

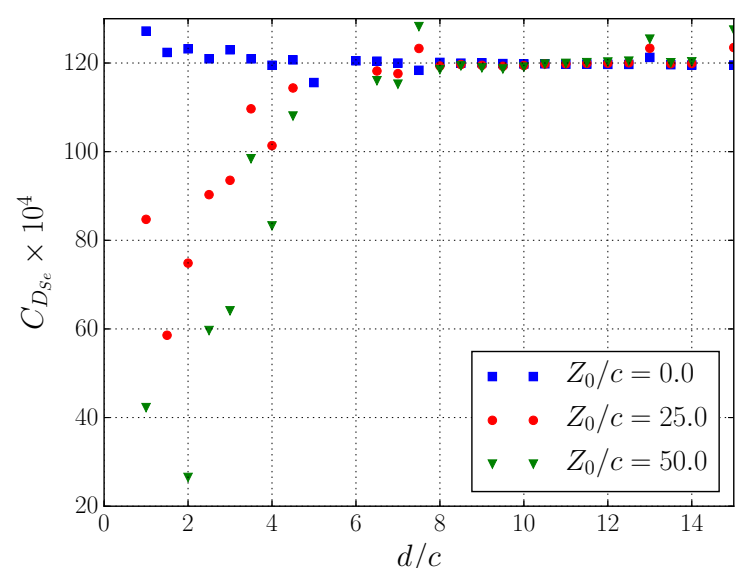

(b) $Z \rightarrow Z+Z_{0}$

Fig. $3 C_{D_{S e}}$ variations for the OAT15A, $M_{\infty}=0.724, R e=3 \times 10^{6}$ and $\alpha=1.15^{\circ}$

The large discrepancies occuring for $d / c=13$ and 15 are attributable to numerical errors caused by the coarsening of the mesh in the computation of the Lamb vector. The next subsection will show that the formula is also sensitive to the size of the control volume.

\section{B. The choice of the integration domain}

\section{Presentation of the problem}

In the derivation of the Lamb-vector-based formulation, the choice of the integration domain surrounding the aircraft can be made arbitrarily. The term $\boldsymbol{F}_{\tau}$ can be dropped as soon as the boundary layer region is completely enclosed in the domain. Therefore, it is of high interest to investigate whether the aerodynamic force breakdown is dependent or not 
on such a choice. Hence, one must assess the contribution of the aerodynamic force provided by an extension of the control volume as sketched in Fig 4

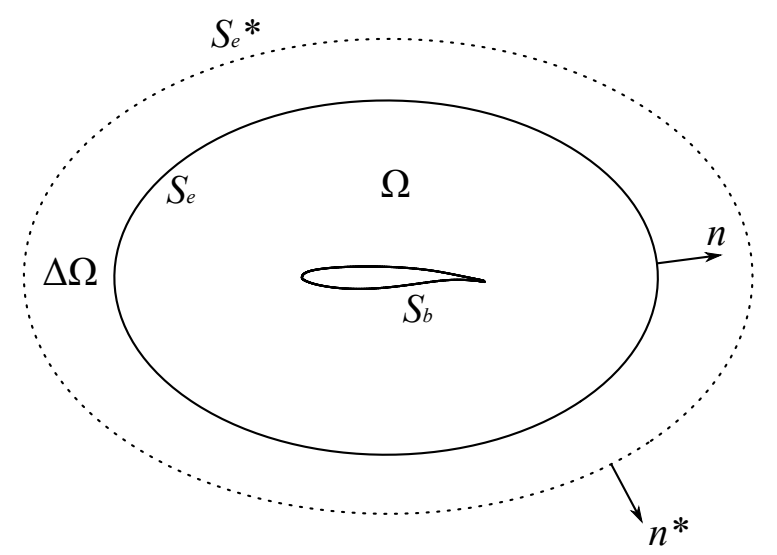

Fig. 4 Extension of the control volume $\Omega$

To do so, one must derive a Lamb-vector-based momentum balance in the extension $\Delta \Omega$ by using the same theoretical developments that led to Eq.(4). In fact, the steady compressible Navier-Stokes equations write:

$$
\rho \boldsymbol{l}+\rho \nabla\left(\frac{u^{2}}{2}\right)=-\nabla p+\nabla \cdot \boldsymbol{\tau}
$$

By taking the curl of Eq. 15 the pressure gradient vanishes such that:

$$
\nabla \times(\rho \boldsymbol{l})+\nabla \rho \times \nabla\left(\frac{u^{2}}{2}\right)=\nabla \times(\nabla \cdot \tau)
$$

The integration on $\Delta \Omega$ along with the application of Eq.A.1 yields the desired Lamb-vector-based momentum balance:

$$
\begin{aligned}
-\int_{\Delta \Omega} \rho \boldsymbol{l} \mathrm{d} v-\int_{\Delta \Omega} \boldsymbol{m}_{\rho} \mathrm{d} v= & -\frac{1}{n-1} \oint_{S_{e}} \boldsymbol{r} \times(\boldsymbol{n} \times \rho \boldsymbol{l}) \mathrm{d} S+\frac{1}{n-1} \oint_{S_{e}^{*}} \boldsymbol{r} \times\left(\boldsymbol{n}^{*} \times \rho \boldsymbol{l}\right) \mathrm{d} S \\
& +\frac{1}{n-1} \oint_{S_{e}} \boldsymbol{r} \times(\boldsymbol{n} \times \nabla \cdot \boldsymbol{\tau}) \mathrm{d} S+\oint_{S_{e}} \boldsymbol{\tau} \cdot \boldsymbol{n} \mathrm{d} S \\
& -\frac{1}{n-1} \oint_{S_{e}^{*}} \boldsymbol{r} \times\left(\boldsymbol{n}^{*} \times \nabla \cdot \boldsymbol{\tau}\right) \mathrm{d} S-\oint_{S_{e}^{*}} \boldsymbol{\tau} \cdot \boldsymbol{n}^{*} \mathrm{~d} S
\end{aligned}
$$

Therefore, it is possible to emphasize the transfer occuring in $\Delta \Omega$ between the reversible part of the force $\boldsymbol{F}_{\rho l}+\boldsymbol{F}_{m_{\rho}}$ and the irreversible part $\boldsymbol{F}_{S_{e}}+\boldsymbol{F}_{\tau}$ while the total force remains constant:

$$
\boldsymbol{F}_{S_{e}}^{*}+\boldsymbol{F}_{\tau}^{*}-\left(\boldsymbol{F}_{S_{e}}+\boldsymbol{F}_{\tau}\right)=-\left(\boldsymbol{F}_{\rho l, \Delta \Omega}+\boldsymbol{F}_{m_{\rho}, \Delta \Omega}\right)
$$




\section{Evolution of the profile drag with the size of the domain}

To illustrate this transfer with a practical case, another numerical computation was performed for transonic cruise flight conditions around the NASA CRM with a Mach number $M_{\infty}=0.85$, a Reynolds number $R e=5 \times 10^{6}$ and a lift coefficient $C_{L}=0.5$. The mesh used in this study is the L4 ${ }^{\prime}$ version from the DPW-5 [45] which is a fine mesh. It is a multiblock grid with $17,252,352$ hexahedral cells. The $y+$ is equal to 0.67 and the far field lies more than 100 reference chord lengths away from the body. This mesh has exactly the same characteristics as the L4 version [46] but has been modified with a wing-twist to match wind-tunnel experiments. The reference chord of the wing is $c_{r e f}=7.00532 \mathrm{~m}$. Convergence was attained and the residuals decreased by $10^{13}$. A grid convergence study on this configuration can be found in Appendix C.

In order to reduce the order of the derivatives involved in the profile drag integral, the irreversible part of the aerodynamic force $\boldsymbol{F}_{S_{e}}+\boldsymbol{F}_{\tau}$ can be expressed in terms of thermodynamic variables by using the steady Crocco-Vazsonyi equation Eq.[19]:

$$
\begin{aligned}
& \rho \boldsymbol{l}-\nabla \cdot \boldsymbol{\tau}=\frac{p}{R} \nabla s-\rho \nabla H \\
& \boldsymbol{F}_{\Delta s}=\boldsymbol{F}_{S_{e}}+\boldsymbol{F}_{\tau}=\boldsymbol{F}_{\Delta s}^{I}+\boldsymbol{F}_{\Delta s}^{I I}+\oint_{S_{e}} \boldsymbol{\tau} \cdot \boldsymbol{n} \mathrm{d} S
\end{aligned}
$$

where

$$
\begin{aligned}
\boldsymbol{F}_{\Delta s}^{I} & =\oint_{S_{e}}\left(\frac{p}{R} \Delta s-\rho \Delta H\right) \boldsymbol{n} \mathrm{d} S \\
\boldsymbol{F}_{\Delta s}^{I I} & =\frac{1}{n-1} \oint_{S_{e}} \boldsymbol{r} \times\left(\boldsymbol{n} \times\left\{\frac{\Delta s}{R} \nabla p-\Delta H \nabla \rho\right\}\right) \mathrm{d} S
\end{aligned}
$$

The profile drag coefficient was then computed in two different ways referred to as $C_{D_{P, l}}$ and $C_{D_{P, \Delta s}}$ and respectively defined by Eq. 24] and 25):

$$
\begin{aligned}
C_{D_{i, l}} & =\frac{2\left(\boldsymbol{F}_{\rho l}+\boldsymbol{F}_{m_{\rho}}\right) \cdot \boldsymbol{e}_{x}}{\rho_{\infty} U_{\infty} S_{r e f}} \\
C_{D_{P, l}} & =\frac{2 \boldsymbol{F}_{S_{e}} \cdot \boldsymbol{e}_{x}}{\rho_{\infty} U_{\infty} S_{r e f}}=C_{D_{S_{e}}} \\
C_{D_{P, \Delta s}} & =\frac{2 \boldsymbol{F}_{\Delta s} \cdot \boldsymbol{e}_{x}}{\rho_{\infty} U_{\infty} S_{r e f}}
\end{aligned}
$$

Fig 5 displays the evolution of the profile drag and lift-induced drag contributions with respect to $d$. The profile drag evolution computed with Eq.25) (yellow triangled curve) is enhanced compared to the original integral Eq. 24). Indeed the evolution of the profile drag is better grasped and the total drag prediction $C_{D_{i, l}}+C_{D_{P, \Delta s}}$ is smoother. Moreover, as it replaces the Lamb vector by the entropy and total enthalpy variations, this equivalent expression avoids numerical 


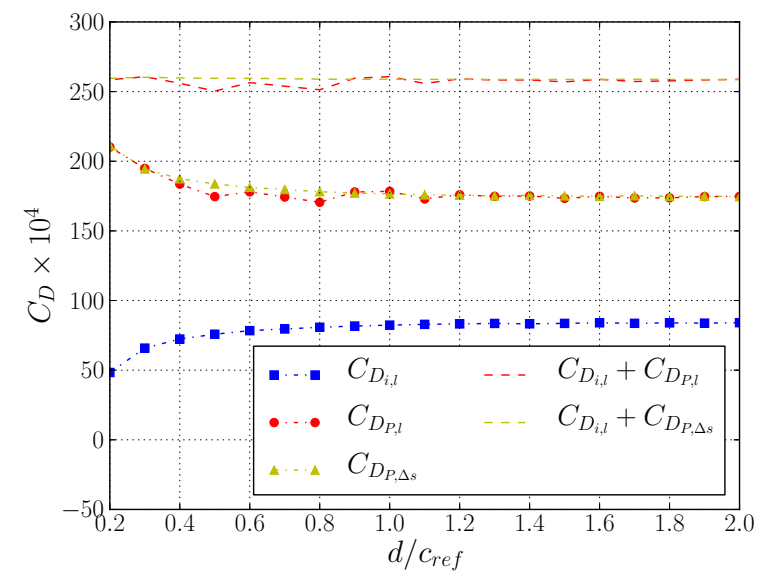

Fig. 5 Drag breakdown evolution with the size of $\Omega$, NASA CRM at $M_{\infty}=0.85, R e=5 \times 10^{6}$ and $C_{L}=0.5$

errors from the computation of the vorticity on ever coarser parts of the grid. It is noteworthy that the lift-induced drag keeps increasing even downstream of the wing trailing edge which supports former studies suggesting that the near wake must be taken into account in the control volume [36, 41, 42]. Only about one reference chord length downstream does the drag breakdown become almost constant which implies that the following condition is progressively verified:

$$
\left(\boldsymbol{F}_{\rho l, \Delta \Omega}+\boldsymbol{F}_{m_{\rho}, \Delta \Omega}\right) \cdot \boldsymbol{e}_{x} \approx 0
$$

It suggests that the Lamb-vector-based drag breakdown can be defined only if the control volume extends far enough in the wake. In the following, it will be shown that the gradual invariance of the breakdown with respect to the size of the integration domain can be explained by the far-field symmetries satisfied by the flow. In fact, as the symmetries progressively establish in the wake, the profile and lift-induced drag respectively decrease and increase (for $0 \leq d / c_{\text {ref }} \leq 1$ on Fig 5 ) until they reach an extremum (for $d / c_{\text {ref }}=1$ on Fig 5 . Further downstream, the lift-induced drag will anyway be transferred to the profile drag because of physical and numerical dissipation which makes it difficult to correctly define the integration domain [40].

\section{The flow symmetries}

\section{A. The three-dimensional wake flow symmetries}

The study of symmetries is widely spread in modern physics since they are often used to shed light on invariance properties. The first symmetries considered here are the symmetries in the far wake of an aircraft. Under steady cruise flight conditions, it is expected that the flow around an aircraft naturally satisfies a symmetry with respect to the $(x, z)$-plane. Further downstream, the velocity defect $u_{x}-U_{\infty}$ caused by the boundary layers and shock waves progressively becomes symmetrical with respect to the $(x, y)$-plane [47] because of the rolling-up of the vortex sheet 
and the viscous diffusion of the wake. Indeed, the rolling-up of the trailing vortex sheet gives birth to a pair of counter-rotating vortices [47. 50] as sketched in Fig,6. From now on, the focus is set on the steady far field [51, 52] where the expansion of the wake and the trailing vortices is assumed to be slow.

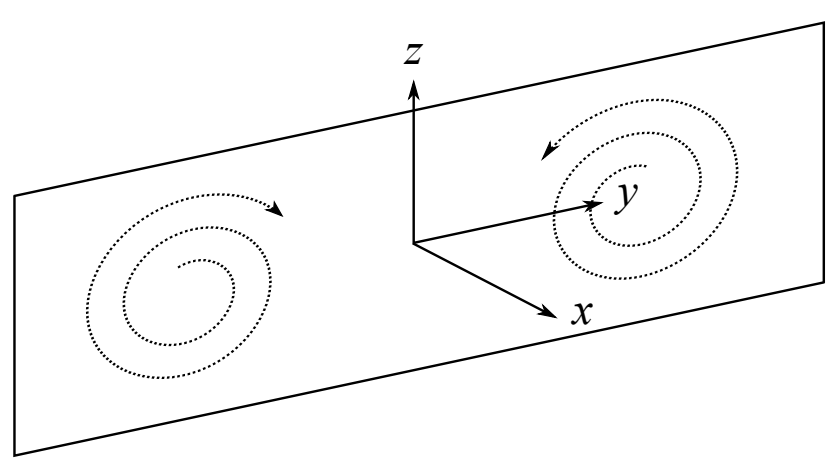

Fig. 6 Counter-rotating vortex pair in the far wake

Thus, far enough downstream, the symmetry properties that are presented below are based on the concentrated dipole [49, 53] sketched in Fig 6 and the following assumptions:

1) The longitudinal velocity distribution $u_{x}$ is symmetrical with respect to the $(x, y)$ and $(x, z)$-planes.

2) The transverse part of the flow field $\left(u_{y}, u_{z}\right)$ consists of a counter-rotating vortex pair.

3) The $x$-derivatives are negligible compared to the $y$ and $z$-derivatives $[3]: \frac{\partial}{\partial x} \ll \frac{\partial}{\partial y}, \frac{\partial}{\partial z}$.

4) The external boundary $S_{e}$ is assumed to be symmetrical with respect to the $(x, y),(x, z)$ and $(y, z)$-planes.

Let $S_{x}, S_{y}$ and $S_{z}$ be defined as follows:

$$
\boldsymbol{S}_{x}=\left(\begin{array}{ccc}
-1 & 0 & 0 \\
0 & 1 & 0 \\
0 & 0 & 1
\end{array}\right), \quad \boldsymbol{S}_{y}=\left(\begin{array}{ccc}
1 & 0 & 0 \\
0 & -1 & 0 \\
0 & 0 & 1
\end{array}\right) \quad \text { and } \quad \boldsymbol{S}_{z}=\left(\begin{array}{ccc}
1 & 0 & 0 \\
0 & 1 & 0 \\
0 & 0 & -1
\end{array}\right)
$$

In the vortical wake, hypotheses 1 and 2 imply that the transverse velocity vector is symmetrical with respect to the $(x, z)$-plane, antisymmetrical with respect to the $(x, y)$-plane and that the density and the kinetic energy are symmetrical with respect to both:

$$
\begin{aligned}
& \boldsymbol{u}(x,-y, z)=\boldsymbol{u}(x, y,-z)=\boldsymbol{S}_{y} \cdot \boldsymbol{u}(x, y, z) \\
& \Phi(x,-y, z)=\Phi(x, y,-z)=\Phi(x, y, z)
\end{aligned}
$$

where $\Phi=\frac{u^{2}}{2}$ or $\rho$. Say that a scalar function $f: x \rightarrow f(x)$ fulfills $f(-x)=f(x)$, therefore its derivative satisfies $f^{\prime}(-x)=-f^{\prime}(x)$. Upon applying this property to the velocity vector field by using Eqs. 28a) and (B.3) (derived in 
Appendix B), it is possible to prove that the vorticity vector field verifies:

$$
\left\{\begin{array}{l}
\omega_{x}(x,-y, z)=-\omega_{x}(x, y, z) \\
\omega_{y}(x,-y, z)=\omega_{y}(x, y, z) \\
\omega_{z}(x,-y, z)=-\omega_{z}(x, y, z)
\end{array}\right.
$$

and

$$
\left\{\begin{array}{l}
\omega_{x}(x, y,-z)=\omega_{x}(x, y, z) \\
\omega_{y}(x, y,-z)=-\omega_{y}(x, y, z)+x \text {-derivatives } \\
\omega_{z}(x, y,-z)=\omega_{z}(x, y, z)+x \text {-derivatives }
\end{array}\right.
$$

As expected from the concentrated dipole model, $\omega_{x}$ is antisymmetrical with respect to the $(x, z)$-plane which is consistent with the counter-rotating tip vortices in the wake. The transverse vorticity is generated by shearing processes inside the boundary layers, $\omega_{y}$ on horizontal surfaces and $\omega_{z}$ on vertical surfaces. Therefore, $\omega_{y}$ and $\omega_{z}$ are expected to be respectively symmetrical and antisymmetrical with respect to the $(x, z)$-plane. Regarding the symmetries across the $(x, y)$-plane, hypothesis 3 is true as soon as the wake evolves slowly further downstream such that the $\mathrm{x}$-derivatives are practically negligible. Consequently, the vorticity field fulfills the following symmetry properties when $S_{e}$ retreats further away:

$$
\omega(x,-y, z)=-\omega(x, y,-z)=-S_{y} \cdot \omega(x, y, z)
$$

Therefore, using Eqs.28a, 28b and 231) and knowing that $\boldsymbol{l}=\boldsymbol{\omega} \times \boldsymbol{u}$, it is possible to derive the symmetry properties of the Lamb vector field in the far wake:

$$
\rho \boldsymbol{l}(x,-y, z)=-\rho \boldsymbol{l}(x, y,-z)=\boldsymbol{S}_{y} \cdot \rho \boldsymbol{l}(x, y, z)
$$

Hypothesis 4 implies that the unit normal is symmetrical with respect to the $(x, y)$ and $(x, z)$-planes. Besides, using Eqs. (28b) and the properties Eqs. B.1) and (B.2) (established in appendix B), one can express the symmetries satisfied by $\nabla \rho, \nabla\left(\frac{u^{2}}{2}\right), \frac{u^{2}}{2} \nabla \rho$ and the unit normal $\boldsymbol{n}$ :

$$
\begin{gathered}
\boldsymbol{f}(x,-y, z)=\boldsymbol{S}_{y} \cdot \boldsymbol{f}(x, y, z) \\
\boldsymbol{f}(x, y,-z)=\boldsymbol{S}_{z} \cdot \boldsymbol{f}(x, y, z) \\
\text { with } \quad \boldsymbol{f}=\nabla \rho, \nabla\left(\frac{u^{2}}{2}\right), \frac{u^{2}}{2} \nabla \rho \text { and } \boldsymbol{n}
\end{gathered}
$$


The gradients are antisymmetrical with respect to the $(x, y)$ and $(x, z)$-planes which is consistent with the fact that $\rho$ and $u^{2} / 2$ are symmetrical. In the end, the symmetry properties derived above yield:

$$
\begin{gathered}
\boldsymbol{f}(x,-y, z)=-\boldsymbol{S}_{y} \cdot \boldsymbol{f}(x, y, z) \\
\text { for } \boldsymbol{f}=\boldsymbol{n} \times \frac{u^{2}}{2} \nabla \rho, \nabla\left(\frac{u^{2}}{2}\right) \times \nabla \rho \text { and } \boldsymbol{n} \times \rho \boldsymbol{l} \\
\boldsymbol{f}(x, y,-z)=-\boldsymbol{S}_{z} \cdot \boldsymbol{f}(x, y, z) \\
\text { for } \boldsymbol{f}=\boldsymbol{n} \times \frac{u^{2}}{2} \nabla \rho \text { and } \nabla\left(\frac{u^{2}}{2}\right) \times \nabla \rho \\
\text { while } \quad(\boldsymbol{n} \times \rho \boldsymbol{l})(x, y,-z)=\rho\left(\begin{array}{c}
-n_{y} l_{z}+n_{z} l_{y} \\
n_{z} l_{x}+n_{x} l_{z} \\
n_{x} l_{y}+n_{y} l_{x}
\end{array}\right)(x, y, z)
\end{gathered}
$$

\section{B. The upstream-downstream symmetry properties}

The study presented above focused on the symmetries satisfied in the wake of an aircraft. Indeed, the Lamb vector field is nonzero only in the boundary layers, in the wake and behind a curved shock wave. On the contrary, $\frac{u^{2}}{2} \nabla \rho$ may well be different from zero in other regions of the flow. For instance, in compressible flows the presence of an obstacle produces a density gradient upstream of the leading edge and as $S_{e}$ extends further away from the body, the density progressively recovers its freestream value. Hence, this section will present a new set of symmetries which is satisfied outside the wake between the upstream and downstream flow domains.

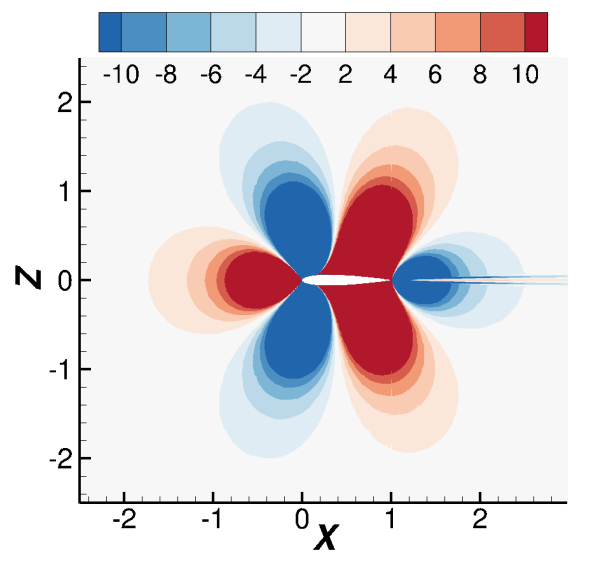

(a) Contour of $\frac{u^{2}}{2} \frac{\partial \rho}{\partial x}$ for $\alpha=0^{\circ}$

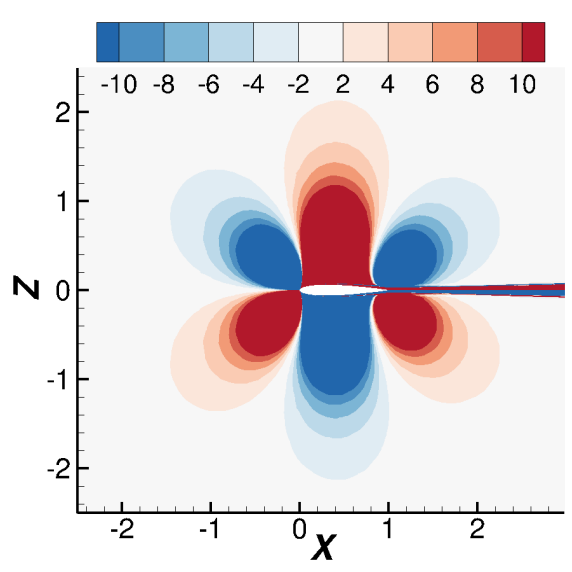

(b) Contour of $\frac{u^{2}}{2} \frac{\partial \rho}{\partial z}$ for $\alpha=0^{\circ}$

Fig. 7 Contours of $\frac{u^{2}}{2} \nabla \rho$ around a NACA0012 airfoil at $R e=3 \times 10^{6}, M_{\infty}=0.4, \alpha=0^{\circ}$

To illustrate this point, numerical computations were performed for a compressible steady viscous flow around 
a NACA0012 airfoil with a Mach number $M_{\infty}=0.4$ and a Reynolds number $R e=3 \times 10^{6}$. The mesh used for the computation contains 76,534 nodes and the far field lies again 50,000 chords away from the body. The computation was converged to a drop in residuals of $10^{13}$. The postprocess of the CFD solution evidenced that the $y+$ is always lower than 1. Fig 7 sketches contours of $\frac{u^{2}}{2} \nabla \rho$ for $\alpha=0^{\circ}$. In this symmetrical case, the flow first undergoes a compression in front of the leading edge which is characterized by a positive $x$-wise density gradient (see Fig 7a). It further accelerates on the pressure and suction sides because of the wall curvature which yields a negative $x$-wise density gradient (expansion). Further downstream the flow faces an adverse pressure gradient and decelerates: it corresponds to the region where the $x$-wise density gradient becomes positive. Downstream of the trailing edge the flow is again expanded and the $x$-wise density gradient becomes negative. In Fig $7 \mathrm{~b}$, on the suction side of the airfoil the flow deceleration upstream of the leading edge decreases with the distance from the wall so that the $z$-wise density gradient is negative. The acceleration occuring downstream is more important close to the wall such that the density increases with $z$ and the $z$-wise density gradient becomes positive. Further, the trailing edge is characterized by a higher pressure which explains why the $z$-wise density gradient becomes negative. The above-described phenomena also occur when $\alpha \neq 0^{\circ}$ but the symmetry observed in the symmetrical case vanishes: indeed, this symmetry breaking is due to the generation of lift. Actually, new symmetry properties appear when seen from afar. It is more obvious in the case of the OAT15A airfoil, where these symmetries are clearly visible outside the wake (see Fig 8 ). The study of the far field on $S_{e}$ fulfilling hypothesis 4 suggests that $\frac{u^{2}}{2} \nabla \rho$ and the unit normal satisfy the following symmetry property with respect to the $(y, z)$-plane:

$$
\begin{aligned}
& f(-x, y, z)=\boldsymbol{S}_{x} \cdot \boldsymbol{f}(x, y, z) \\
& \text { for } \boldsymbol{f}=\frac{u^{2}}{2} \nabla \rho \text { and } \boldsymbol{n}
\end{aligned}
$$

such that, outside the wake:

$$
\begin{gathered}
f(-x, y, z)=-\boldsymbol{S}_{x} \cdot \boldsymbol{f}(x, y, z) \\
\text { for } \boldsymbol{f}=\boldsymbol{n} \times \frac{u^{2}}{2} \nabla \rho \text { and } \nabla\left(\frac{u^{2}}{2}\right) \times \nabla \rho
\end{gathered}
$$

The Lamb-vector-based force breakdown is inherently dependent on the size of the control volume and the choice of the reference point as illustrated in section III. In the present section, it has been shown that a compressible steady viscous flow around an obstacle progressively respects symmetries in the far field. In the context of those symmetries, the next section will explain how the Lamb-vector-based formulation (4) gradually becomes invariant. A new formula which guarantees the invariance with respect to the reference point even in the near field will eventually be established. 


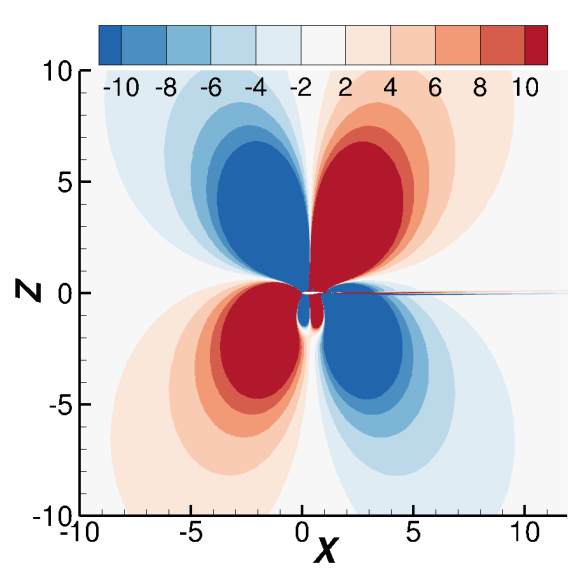

(a) Contour of $\frac{u^{2}}{2} \frac{\partial \rho}{\partial x}$

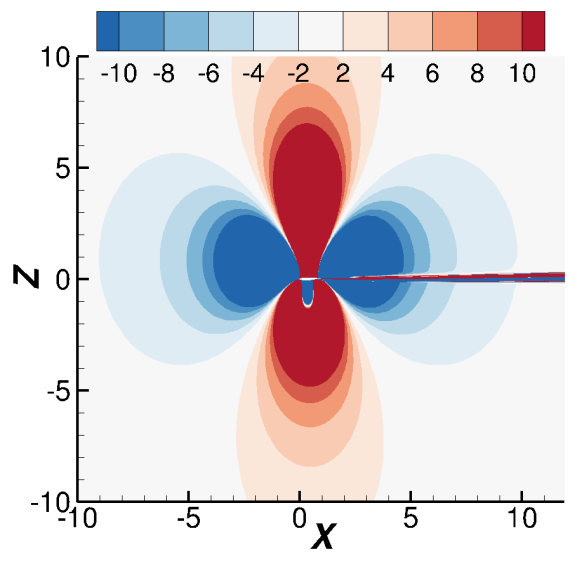

(b) Contour of $\frac{u^{2}}{2} \frac{\partial \rho}{\partial z}$

Fig. 8 Contours of $\frac{u^{2}}{2} \nabla \rho$ around an OAT15A airfoil at $R e=3 \times 10^{6}, M_{\infty}=0.724$ and $\alpha=1.15^{\circ}$

\section{An invariant force breakdown}

\section{A. Far-field symmetries and invariance}

\section{Far-field invariance with respect to the reference point}

This paragraph explains how the Lamb-vector-based aerodynamic force breakdown naturally becomes independent of the reference point when $S_{e}$ retreats to infinity. Let $S_{w}$ be the portion of $S_{e}$ crossing the wake. Using the wake flow symmetries Eqs. 34a and 34b and limiting the integration to the wake on $S_{w}$ :

$$
\begin{aligned}
\int_{S_{w}}\left(\boldsymbol{n} \times \frac{u^{2}}{2} \nabla \rho\right) \mathrm{d} S & =\int_{S_{w}, y \geq 0}\left(\boldsymbol{n} \times \frac{u^{2}}{2} \nabla \rho\right) \mathrm{d} S+\int_{S_{w}, y \leq 0}\left(\boldsymbol{n} \times \frac{u^{2}}{2} \nabla \rho\right) \mathrm{d} S \\
& =\left(\mathbf{1}-\boldsymbol{S}_{y}\right) \cdot \int_{S_{w}, y \geq 0}\left(\boldsymbol{n} \times \frac{u^{2}}{2} \nabla \rho\right) \mathrm{d} S \\
& =\underbrace{\left(\mathbf{1}-\boldsymbol{S}_{y}\right) \cdot\left(\mathbf{1}-\boldsymbol{S}_{z}\right)}_{=0} \cdot \int_{S_{w}, y, z \geq 0}\left(\boldsymbol{n} \times \frac{u^{2}}{2} \nabla \rho\right) \mathrm{d} S=\mathbf{0}
\end{aligned}
$$

Nevertheless,

$$
\int_{S_{w}}(\boldsymbol{n} \times \rho \boldsymbol{l}) \mathrm{d} S=4\left(\int_{S_{w}, y, z \geq 0} \rho n_{z} l_{x} \mathrm{~d} S\right) \boldsymbol{e}_{y}
$$

Thus, according to Eq. 38) there is no evidence that $\int_{S_{w}}(\boldsymbol{n} \times \rho \boldsymbol{l}) \mathrm{d} S=\mathbf{0}$. However, in the far wake the velocity field can be approximated as $\boldsymbol{u}=\left(U_{\infty}+u_{x}^{\prime}\right) \boldsymbol{e}_{x}+u_{y} \boldsymbol{e}_{y}+u_{z} \boldsymbol{e}_{z}$ such that $\left|\frac{u_{x}^{\prime}}{U_{\infty}}\right|,\left|\frac{u_{y}}{U_{\infty}}\right|,\left|\frac{u_{z}}{U_{\infty}}\right| \ll 1$. In this case, the Lamb vector 


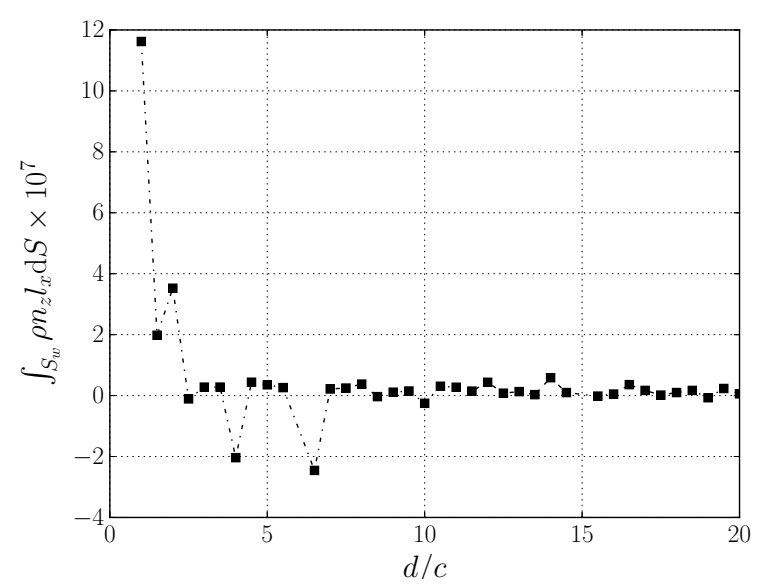

Fig. 9 Evolution of the integral Eq. 38 in the wake

consists of a first order and a second order term:

$$
\boldsymbol{l}=\boldsymbol{l}_{1}+\boldsymbol{l}_{2}=\underbrace{\left(\begin{array}{c}
0 \\
\omega_{z} U_{\infty} \\
-\omega_{y} U_{\infty}
\end{array}\right)}_{\text {first order }}+\underbrace{\left(\begin{array}{c}
\omega_{y} u_{z}-\omega_{z} u_{y} \\
\omega_{z} u_{x}^{\prime}-\omega_{x} u_{z} \\
\omega_{x} u_{y}-\omega_{y} u_{x}^{\prime}
\end{array}\right)}_{\text {second order }}
$$

Besides, in the far wake the direction of the flow is almost parallel to that of the freestream and the region where the Lamb vector is nonzero coincides with a portion of $S_{w}$ where $\boldsymbol{n} \approx \boldsymbol{e}_{x}$. Therefore, at first order $l_{x} \ll l_{y}, l_{z}$ (see [44]) and $n_{y}, n_{z} \ll n_{x}$ such that the integral Eq. (38) becomes negligible (of the order of magnitude $10^{-7}$ as shown in Fig 9 ) and the condition Eq. (14) is progressively verified.

Using now the upstream-downstream symmetry Eq.(36), the following condition is derived:

$$
\int_{S_{e}-S_{w}}\left(\boldsymbol{n} \times \frac{u^{2}}{2} \nabla \rho\right) \mathrm{d} S=\underbrace{\left(\mathbf{1}-\boldsymbol{S}_{x}\right) \cdot\left(\mathbf{1}-\boldsymbol{S}_{y}\right)}_{=0} \cdot \int_{S_{e}-S_{w}, x, y \geq 0}\left(\boldsymbol{n} \times \frac{u^{2}}{2} \nabla \rho\right) \mathrm{d} S=\mathbf{0}
$$

Therefore, the discussion on Eq. (38) and the results given by Eqs. (37) and (40) suggest that the additional terms with $\boldsymbol{r}_{0}$ in Eq.(11) vanish in the far field, invariance is achieved and the force breakdown remains unchanged with a shift in the reference point:

$$
\begin{aligned}
& \lim _{d \rightarrow \infty} \oint_{S_{e}} \boldsymbol{n} \times \rho \boldsymbol{l} \mathrm{d} S=\mathbf{0} \\
& \lim _{d \rightarrow \infty} \oint_{S_{e}} \boldsymbol{n} \times \frac{u^{2}}{2} \nabla \rho \mathrm{d} S=\mathbf{0}
\end{aligned}
$$


In conclusion, it has been shown that as soon as $S_{e}$ stands far enough from the body, the flow quantities $\rho l$ and $\frac{u^{2}}{2} \nabla \rho$ satisfy proper far-field symmetries which eliminate the sensitivity of the Lamb-vector-based force breakdown to the choice of the reference point.

\section{Far-field invariance with respect to the size of the control volume}

This paragraph examines how the far-field flow symmetries impact the evolution of the force breakdown as $S_{e}$ extends further away from the body. The domain extension $\Delta \Omega$ is supposed to lie in the far field where the flow satisfies

all the symmetry properties. Let $\Delta \Omega_{w}$ be the part of $\Delta \Omega$ crossing the wake. The wake flow symmetries Eq. 32. satisfied by the Lamb vector field explain why the vortex force contribution vanishes:

$$
\boldsymbol{F}_{\rho l, \Delta \Omega}=\int_{\Delta \Omega_{w}} \rho \boldsymbol{l} \mathrm{d} v=\underbrace{\left(\mathbf{1}+\boldsymbol{S}_{y}\right) \cdot\left(\mathbf{1}-\boldsymbol{S}_{y}\right)}_{=0} \cdot \int_{\Delta \Omega_{w}, y, z \geq 0} \rho \boldsymbol{l} \mathrm{d} v=\mathbf{0}
$$

Unlike $\boldsymbol{F}_{\rho l}$, the drag contribution of $\boldsymbol{F}_{m_{\rho}}$ quickly stabilizes in the far field as observed in Fig 10 Besides in the near wake, where the symmetries are not fulfilled yet, the lift-induced drag variations are mostly attributable to $\boldsymbol{F}_{\rho l}$. Further downstream $\left(d / c_{\text {ref }} \geq 1\right), \rho \boldsymbol{l}$ does not contribute anymore to the force once it satisfies the wake flow symmetries as stated by Eq.43]. The lift-induced drag then reaches a constant maximum value before the coarsening of the grid slowly dissipates it into profile drag [54]. Consequently, the near wake must be accounted for in the control volume when using the Lamb-vector-based aerodynamic force breakdown so that the far-field symmetries are satisfied and the different contributors have converged.

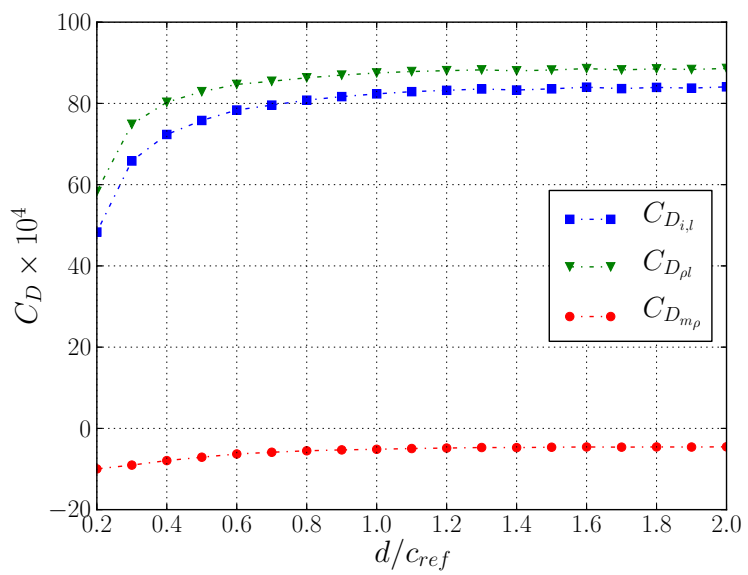

Fig. 10 Evolution of the different lift-induced drag contributors with the size of $\Omega$, NASA CRM at $M_{\infty}=0.85$, $R e=5 \times 10^{6}$ and $C_{L}=0.5$ 


\section{B. Definition of the reference-point-invariant aerodynamic force breakdown}

\section{Principle}

The study of the flow symmetries highlighted the mechanisms that bring the aerodynamic force decomposition to become independent of the reference point in the far field. Therefore, the control volume must be chosen such that the external boundary $S_{e}$ is placed in the region where the symmetries are fulfilled. Unfortunately, this implies that the grid on which the surface integral is computed becomes ever coarser as the size of the domain is increased. Thus finding a method that would make the formulation independent of the reference point everywhere turns out to be crucial.

To do so, one must define new vectors based on the physical fields $\rho \boldsymbol{l}$ and $\frac{u^{2}}{2} \nabla \rho$ such that the two following conditions are satisfied:

1) They must cancel the additional terms with $\boldsymbol{r}_{0}$ appearing in Eqs. 99 and $(10)$ for any size of $\Omega$.

2) Upon integration, they must yield to the same force contribution as the initial vector fields in Eqs.(6) and (7). The first condition implies that the symmetry properties presented above must be verified not only in the far field but everywhere in the fluid. This is done by decomposing the vectors in a part which satisfies those symmetries and another part which is expected to vanish when $S_{e} \rightarrow \infty$. Let us first explain the principle with a 1D example. Let $\phi$ be a function of $\xi$ : then $\phi$ is uniquely decomposed as the sum of a symmetrical part $\phi_{+}$and an antisymmetrical part $\phi_{-}$as follows:

$$
\phi_{+}(\xi)=\frac{1}{2}(\phi(\xi)+\phi(-\xi)) \quad \text { and } \quad \phi_{-}(\xi)=\frac{1}{2}(\phi(\xi)-\phi(-\xi))
$$

The same decomposition will now be applied to the components of the physical vector fields $\rho l$ and $\frac{u^{2}}{2} \nabla \rho$ with respect to the three coordinates $(x, y, z)$ in order to build new vectors that satisfy the symmetry properties derived earlier.

\section{Definition of an invariant $F_{S_{e}}$}

In the previous sections, the Lamb vector was shown to fulfill the symmetry properties Eq. 32 ] in the far wake. From now on, only the transverse part $\left(l_{y}, l_{z}\right)$ of the Lamb vector will be considered as $l_{x}$ is not involved in the computation of the profile drag and can be neglected in the far wake. This vector is denoted $l_{\perp}=\left(\begin{array}{lll}0 & l_{y} & l_{z}\end{array}\right)^{T}$ and satisfies the symmetry properties Eqs. $33 \mathrm{a}$ and $33 \mathrm{~b}$ in the far wake.

In the near wake these symmetries can be enforced upon defining a new transverse Lamb vector $\rho \boldsymbol{l}_{\perp, y z-}$ which 
satisfies the symmetries everywhere. Starting by the $(x, z)$-plane symmetry, one can define $\rho \boldsymbol{l}_{\perp, y-}$ as follows:

$$
\begin{aligned}
\rho \boldsymbol{l}_{\perp, y-}(x, y, z) & =\frac{1}{2}\left(\rho \boldsymbol{l}_{\perp}(x, y, z)+\boldsymbol{S}_{y} \cdot \rho \boldsymbol{l}_{\perp}(x,-y, z)\right) \\
& =\frac{1}{2}\left(\begin{array}{c}
0 \\
l_{y}(x, y, z)-l_{y}(x,-y, z) \\
l_{z}(x, y, z)+l_{z}(x,-y, z)
\end{array}\right)
\end{aligned}
$$

Therefore, it is clear that $\rho \boldsymbol{l}_{\perp, y-}$ satisfies Eq. 33a). In order to fulfill Eqs. 33a and (33b), $\rho \boldsymbol{l}_{\perp, y z-}$ must be defined as follows:

$$
\begin{aligned}
\rho \boldsymbol{l}_{\perp, y z-}(x, y, z) & =\frac{1}{2}\left(\rho \boldsymbol{l}_{\perp, y-}(x, y, z)+\boldsymbol{S}_{z} \cdot \rho \boldsymbol{l}_{\perp, y-}(x, y,-z)\right) \\
& =\frac{1}{4}\left(\rho \boldsymbol{l}_{\perp}(x, y, z)+\boldsymbol{S}_{y} \cdot \rho \boldsymbol{l}_{\perp}(x,-y, z)\right. \\
& \left.+\boldsymbol{S}_{z} \cdot \rho \boldsymbol{l}_{\perp}(x, y,-z)+\boldsymbol{S}_{y z} \cdot \rho \boldsymbol{l}_{\perp}(x,-y,-z)\right)
\end{aligned}
$$

where $S_{y z}=S_{y} \cdot S_{z}$. In order to comply with the fourth hypothesis of section IV.B, one must integrate this vector field on a surface which satisfies symmetries such that the unit normal fulfills the properties defined in Eqs. (33a), 33b) and (35). Thus it is possible to show that $\boldsymbol{n} \times \rho \boldsymbol{l}_{\perp, y z^{-}}$satisfies the symmetries Eqs. 34a and (34b). It implies that the additional term appearing in Eq.99 is exactly zero everywhere and the first condition is satisfied:

$$
\begin{aligned}
\oint_{S_{e}}\left(\boldsymbol{n} \times \rho \boldsymbol{l}_{\perp, y z^{-}}\right) \mathrm{d} S & =\int_{S_{e}, y \geq 0}\left(\boldsymbol{n} \times \rho \boldsymbol{l}_{\perp, y z^{-}}\right) \mathrm{d} S+\int_{S_{e}, y \leq 0}\left(\boldsymbol{n} \times \rho \boldsymbol{l}_{\perp, y z^{-}}\right) \mathrm{d} S \\
& =\left(\mathbf{1}-\boldsymbol{S}_{y}\right) \cdot \int_{S_{e}, y \geq 0}\left(\boldsymbol{n} \times \rho \boldsymbol{l}_{\perp, y z^{-}}\right) \mathrm{d} S \\
& =\underbrace{\left(\mathbf{1}-\boldsymbol{S}_{y}\right) \cdot\left(\mathbf{1}-\boldsymbol{S}_{z}\right)}_{=0} \cdot \int_{S_{e}, y, z \geq 0}\left(\boldsymbol{n} \times \rho \boldsymbol{l}_{\perp, y z^{-}}\right) \mathrm{d} S=\mathbf{0}
\end{aligned}
$$

Morevoer, upon proceeding to the variable shifts $y^{\prime}=-y$ and $z^{\prime}=-z$ in the integration, it is possible to express the corresponding force contribution as follows:

$$
\begin{aligned}
-\frac{1}{n-1} \oint_{S_{e}} \boldsymbol{r} \times\left(\boldsymbol{n} \times \rho \boldsymbol{l}_{\perp, y z-}\right) \mathrm{d} S & =-\frac{1}{4(n-1)} \oint_{S_{e}}\left\{\boldsymbol{r} \times\left(\boldsymbol{n} \times \rho \boldsymbol{l}_{\perp}\right)\right. \\
& +\boldsymbol{S}_{y} \cdot \boldsymbol{r} \times\left(\boldsymbol{S}_{y} \cdot \boldsymbol{n} \times \boldsymbol{S}_{y} \cdot \rho \boldsymbol{l}_{\perp}\right) \\
& +\boldsymbol{S}_{z} \cdot \boldsymbol{r} \times\left(\boldsymbol{S}_{z} \cdot \boldsymbol{n} \times \boldsymbol{S}_{z} \cdot \rho \boldsymbol{l}_{\perp}\right) \\
& \left.+\boldsymbol{S}_{y z} \cdot \boldsymbol{r} \times\left(\boldsymbol{S}_{y z} \cdot \boldsymbol{n} \times \boldsymbol{S}_{y z} \cdot \rho \boldsymbol{l}_{\perp}\right)\right\} \mathrm{d} S
\end{aligned}
$$


Let now $S$ be any of the symmetry matrices defined above. It is straight forward to notice that $\boldsymbol{S}^{T} \cdot \boldsymbol{S}=\mathbf{1}$. Moreover, for any vectors $\boldsymbol{a}$ and $\boldsymbol{b}, \boldsymbol{a} \cdot \boldsymbol{b}=\boldsymbol{a}^{T} \boldsymbol{b}$. Thus, by using the rule of the double cross product it is possible to show that

$$
\begin{aligned}
-\frac{1}{n-1} \oint_{S_{e}} \boldsymbol{r} \times\left(\boldsymbol{n} \times \rho \boldsymbol{l}_{\perp, y z-}\right) \mathrm{d} S & =-\frac{\mathbf{1}+\boldsymbol{S}_{y}+\boldsymbol{S}_{z}+\boldsymbol{S}_{y z}}{4(n-1)} \cdot \oint_{S_{e}} \boldsymbol{r} \times\left(\boldsymbol{n} \times \rho \boldsymbol{l}_{\perp}\right) \mathrm{d} S \\
& =-\frac{1}{(n-1)}\left(\begin{array}{ccc}
1 & 0 & 0 \\
0 & 0 & 0 \\
0 & 0 & 0
\end{array}\right) \oint_{S_{e}} \boldsymbol{r} \times\left(\boldsymbol{n} \times \rho \boldsymbol{l}_{\perp}\right) \mathrm{d} S \\
& =\left(\boldsymbol{e}_{x} \cdot \boldsymbol{F}_{S_{e}}\right) \boldsymbol{e}_{x}
\end{aligned}
$$

Finally, the second condition is recovered and $\rho \boldsymbol{l}_{\perp, y z_{-}}$enables to compute the same profile drag as the one provided by $\boldsymbol{F}_{S_{e}}$ but no lift. As initially wanted, this definition is independent of the reference point.

\section{Definition of an invariant $F_{m_{\rho}}$}

The definition of the invariant version of $\boldsymbol{F}_{m_{\rho}}$ is based on the equivalent form 50 for which Eq. A.1 has been used. Only the surface integral will be addressed as the position vector is not present in the second one.

$$
\boldsymbol{F}_{m_{\rho}}=\frac{1}{n-1} \oint_{S_{e}} \boldsymbol{r} \times\left(\boldsymbol{n} \times \frac{u^{2}}{2} \nabla \rho\right) \mathrm{d} S+\int_{\Omega} \frac{u^{2}}{2} \nabla \rho \mathrm{d} v
$$

Given that $\frac{u^{2}}{2} \nabla \rho$ satisfies the symmetry properties defined by Eqs. 33a and (33b) in the far wake (the same as $\left.\rho \boldsymbol{l}_{\perp}\right)$, it is possible to define $\frac{u^{2}}{2} \nabla \rho_{y z-}$ using Eq.46 and replacing $\rho \boldsymbol{l}_{\perp}$ by $\frac{u^{2}}{2} \nabla \rho$ such that the additional term appearing in Eq. 10 ) is zero and the corresponding force contribution yields a drag component and no lift:

$$
\begin{aligned}
& \oint_{S_{e}}\left(\boldsymbol{n} \times \frac{u^{2}}{2} \nabla \rho_{y z^{-}}\right) \mathrm{d} S=\mathbf{0} \\
& \oint_{S_{e}} \boldsymbol{r} \times\left(\boldsymbol{n} \times \frac{u^{2}}{2} \nabla \rho_{y z^{-}}\right) \mathrm{d} S=\left(\boldsymbol{e}_{x} \cdot \oint_{S_{e}} \boldsymbol{r} \times\left(\boldsymbol{n} \times \frac{u^{2}}{2} \nabla \rho\right) \mathrm{d} S\right) \boldsymbol{e}_{x}
\end{aligned}
$$

In order to recover the lift component, one must consider the symmetry properties outside of the wake, namely the upstream-downstream symmetries. Indeed, outside of the wake, $\frac{u^{2}}{2} \nabla \rho$ satisfies Eqs. 33a and (35). This time it is possible to define $\frac{u^{2}}{2} \nabla \rho_{x y-}$ as follows:

$$
\begin{aligned}
\frac{u^{2}}{2} \nabla \rho_{x y-}(x, y, z) & =\frac{1}{4}\left(\frac{u^{2}}{2} \nabla \rho(x, y, z)+S_{x} \cdot \frac{u^{2}}{2} \nabla \rho(-x, y, z)\right. \\
& \left.+S_{y} \cdot \frac{u^{2}}{2} \nabla \rho(x,-y, z)+S_{x y} \cdot \frac{u^{2}}{2} \nabla \rho(-x,-y, z)\right)
\end{aligned}
$$


where $S_{x y}=S_{x} \cdot S_{y}$. Once again, the additional term appearing in Eq. (10) is zero and the corresponding force contribution yields a lift component and no drag:

$$
\begin{aligned}
& \oint_{S_{e}}\left(\boldsymbol{n} \times \frac{u^{2}}{2} \nabla \rho_{x y-}\right) \mathrm{d} S=\mathbf{0} \\
& \oint_{S_{e}} \boldsymbol{r} \times\left(\boldsymbol{n} \times \frac{u^{2}}{2} \nabla \rho_{x y-}\right) \mathrm{d} S=\left(\boldsymbol{e}_{z} \cdot \oint_{S_{e}} \boldsymbol{r} \times\left(\boldsymbol{n} \times \frac{u^{2}}{2} \nabla \rho\right) \mathrm{d} S\right) \boldsymbol{e}_{z}
\end{aligned}
$$

Finally, upon integrating $\frac{u^{2}}{2} \nabla \rho_{y z-}$ for the drag component and $\frac{u^{2}}{2} \nabla \rho_{x y-}$ for the lift component, the surface integral involved in the term $\boldsymbol{F}_{m_{\rho}}$ becomes completely independent of the reference point.

In the end, the force is reconstructed by means of vectors verifying the far-field symmetry properties everywhere in the control volume such that a shift in the reference point does not change the contribution to the total force. The next section will present some numerical results based on this novel invariant Lamb-vector-based force breakdown.

\section{Analysis of the results}

This section proposes two equivalent invariant formulations based on the Lamb vector that guarantee the invariance with respect to choice of the reference point. Although the external boundary $S_{e}$ has been assumed symmetrical, the aforementioned method may also apply to non-symmetrical surfaces by enforcing the symmetry properties on the unit normal $\boldsymbol{n}$. The reference-point-invariant Lamb-vector-based aerodynamic force decomposition is then defined as follows:

$$
\begin{aligned}
D_{i} & =\boldsymbol{e}_{x} \cdot\left\{\frac{1}{n-1} \oint_{S_{e}} \boldsymbol{r} \times\left(\boldsymbol{n} \times \frac{u^{2}}{2} \nabla \rho_{y z^{-}}\right) \mathrm{d} S-\int_{\Omega}\left(\rho \boldsymbol{l}-\frac{u^{2}}{2} \nabla \rho\right) \mathrm{d} v\right\} \\
D_{S_{e}} & =\boldsymbol{e}_{x} \cdot\left\{-\frac{1}{n-1} \oint_{S_{e}} \boldsymbol{r} \times\left(\boldsymbol{n} \times \rho \boldsymbol{l}_{\perp, y z^{-}}\right) \mathrm{d} S\right\} \\
L & =\boldsymbol{e}_{z} \cdot\left\{\frac{1}{n-1} \oint_{S_{e}} \boldsymbol{r} \times\left(\boldsymbol{n} \times \frac{u^{2}}{2} \nabla \rho_{x y-}\right) \mathrm{d} S-\int_{\Omega}\left(\rho \boldsymbol{l}-\frac{u^{2}}{2} \nabla \rho\right) \mathrm{d} v\right\}
\end{aligned}
$$

Fig 11 plots the profile drag computed by the new definition for the two-dimensional transonic flow and must be compared to Fig 3 the curves corresponding to the different shifts in reference point match perfectly which suggests that the new integral is definitely independent of the reference point. Additional results concerning the invariance of $\boldsymbol{F}_{m_{\rho}}$ in two-dimensional flows can be found in Ref.[44].

The three-dimensional analysis was carried out on the NASA CRM for a Mach number $M_{\infty}=0.85$, a Reynolds number $R e=5 \times 10^{6}$ and a lift coefficient $C_{L}=0.5$. Some numerical issues might occur when computing the lift and lift-induced drag respectively defined by Eq. 587) and Eq.56) (see Figs 13 and 14). Therefore, the invariant form of the term $\boldsymbol{F}_{m_{\rho}}$ may also be computed using the volume integral Eq. (6) for which the symmetries are applied on $\nabla\left(\frac{u^{2}}{2}\right) \times \nabla \rho$. 


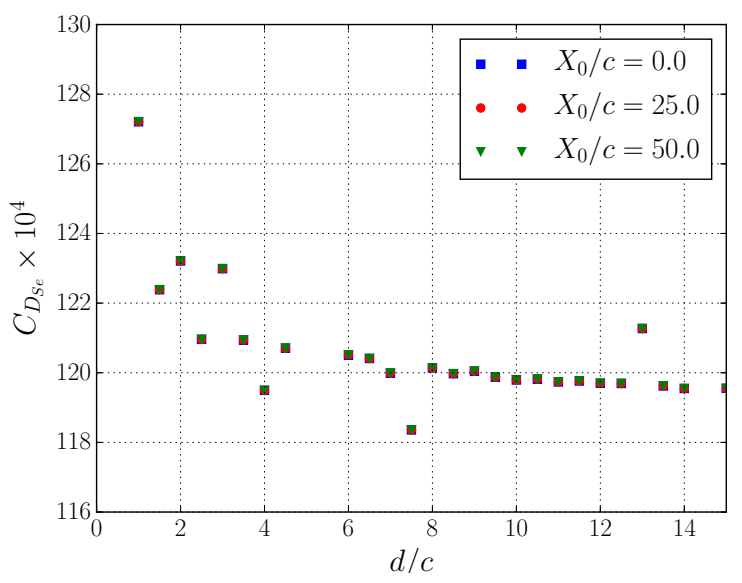

(a) $X \rightarrow X+X_{0}$

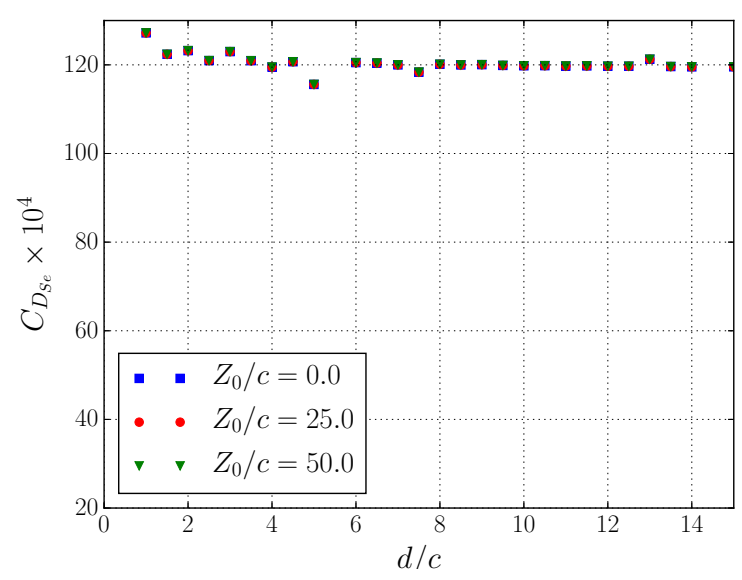

(b) $Z \rightarrow Z+Z_{0}$

Fig. $11 C_{D_{S_{e}}}$ invariance for the OAT15A airfoil, $M_{\infty}=0.724, R e=3 \times 10^{6}$ and $\alpha=1.15^{\circ}$

The lift and the lift-induced drag are then given by:

$$
\begin{array}{r}
D_{i}=\boldsymbol{e}_{x} \cdot\left\{-\int_{\Omega} \rho \boldsymbol{l} \mathrm{d} v+\frac{1}{n-1} \int_{\Omega} \boldsymbol{r} \times\left(\nabla\left(\frac{u^{2}}{2}\right) \times \nabla \rho\right)_{y z-} \mathrm{d} v\right\} \\
L=\boldsymbol{e}_{z} \cdot\left\{-\int_{\Omega} \rho \boldsymbol{l} \mathrm{d} v+\frac{1}{n-1} \int_{\Omega} \boldsymbol{r} \times\left(\nabla\left(\frac{u^{2}}{2}\right) \times \nabla \rho\right)_{x y-} \mathrm{d} v\right\}
\end{array}
$$

Figs 12, 13 and 14 respectively show the computed $C_{D_{S_{e}}}, C_{D_{m_{\rho}}}$ and $C_{L_{m_{\rho}}}$ on the NASA CRM. The results obtained confirm the invariance of the new formulation for three-dimensional flows.

The profile drag was expected to grow as the size of the domain increases. Indeed, in three-dimensional flows the physical viscous diffusion and the numerical dissipation are responsible for the transfer of the drag from the lift-induced to the profile contribution while the total drag remains constant [40, 54]. Schmitt and Destarac [54] had formerly applied a correction based on Oswatitsch formula to compute the profile drag resulting from this transfer. On the contrary it slowly diminishes even far away from the body (see Fig 12). It can be explained by the great resolution of the L4' grid used in this study for which the transfer resulting from the coarsening will occur further downstream in the wake. 


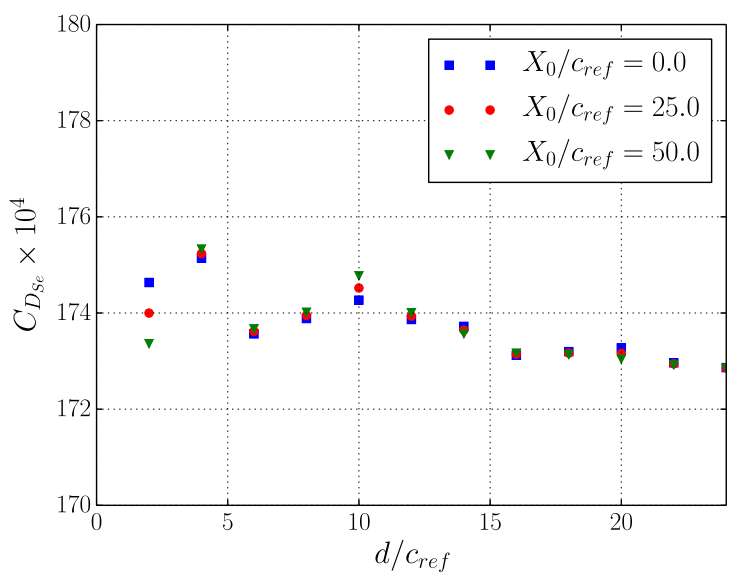

(a) $X \rightarrow X+X_{0}$, old formula

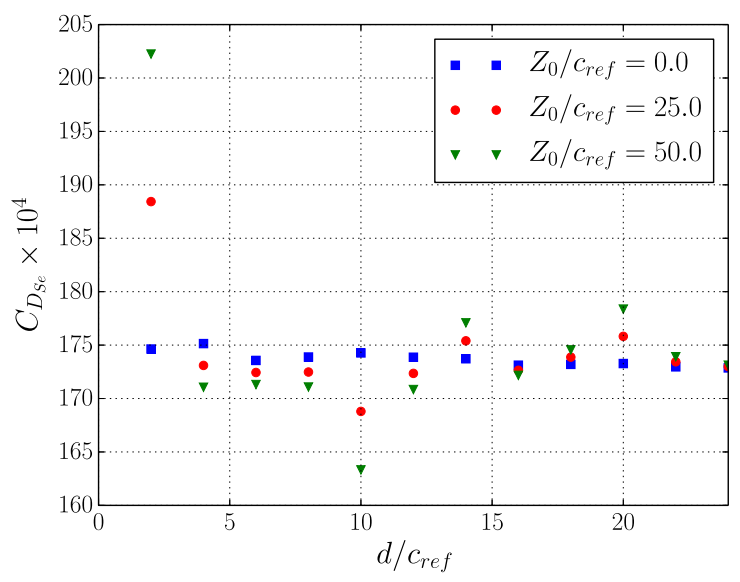

(c) $Z \rightarrow Z+Z_{0}$, old formula

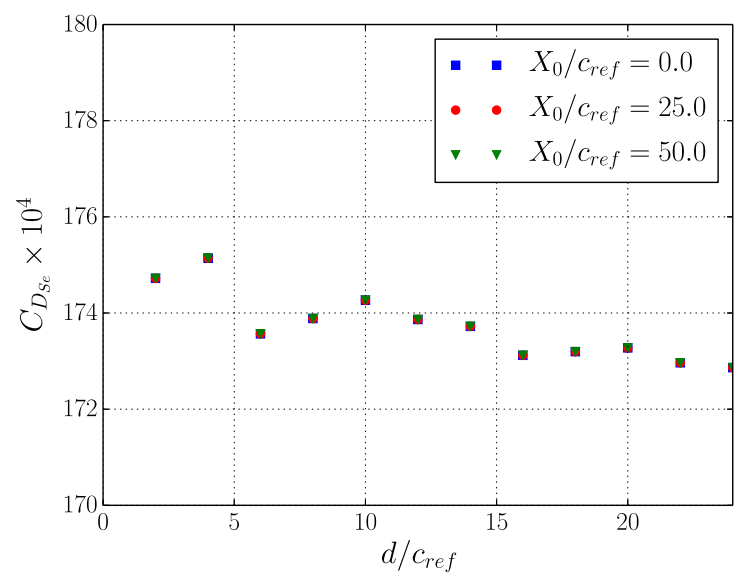

(b) $X \rightarrow X+X_{0}$, new formula

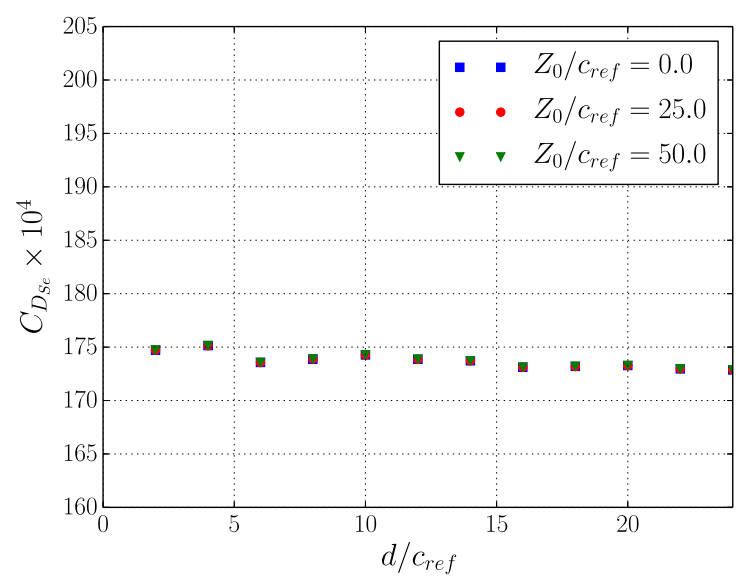

(d) $Z \rightarrow Z+Z_{0}$, new formula

Fig. $12 C_{D_{S_{e}}}$ evolutions for the NASA CRM, $M_{\infty}=0.85, R e=5 \times 10^{6}$ and $C_{L}=0.5$ 


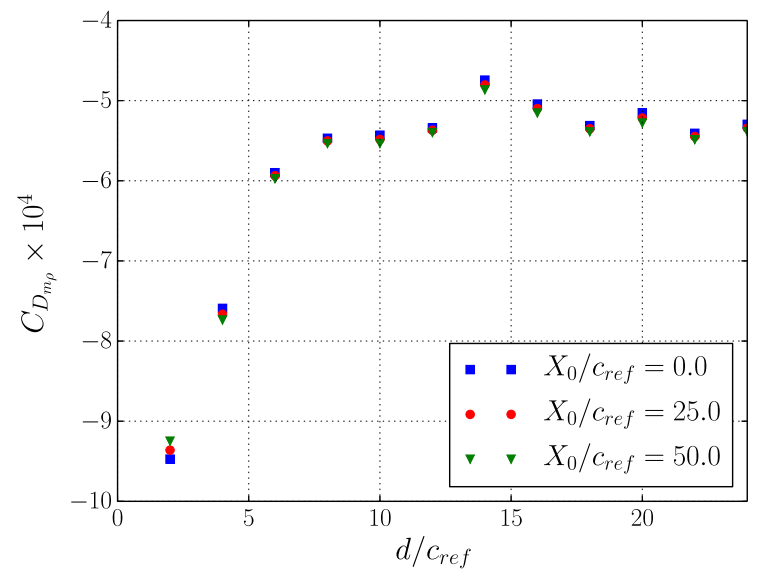

(a) $X \rightarrow X+X_{0}$, old formula

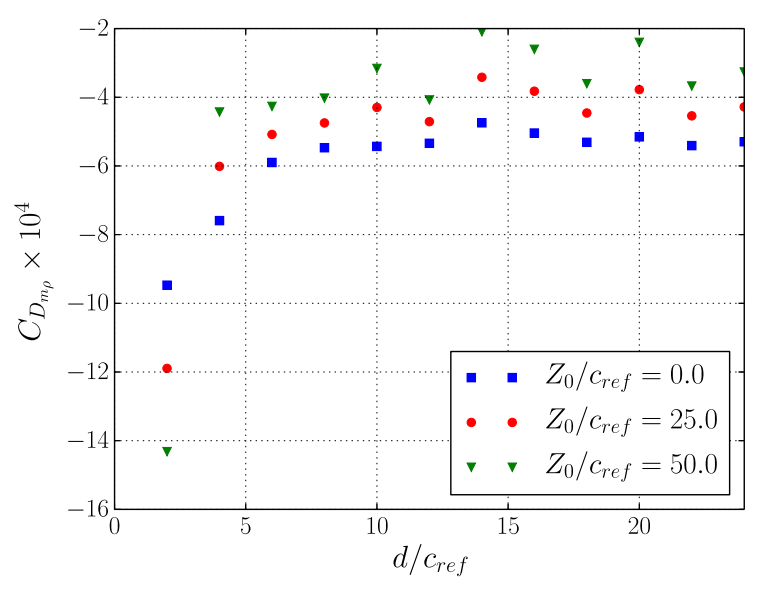

(c) $Z \rightarrow Z+Z_{0}$, old formula

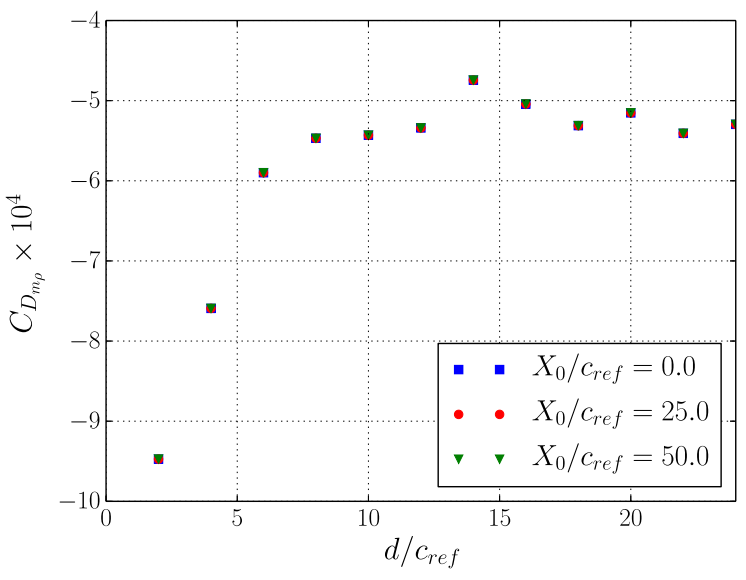

(b) $X \rightarrow X+X_{0}$, new formula

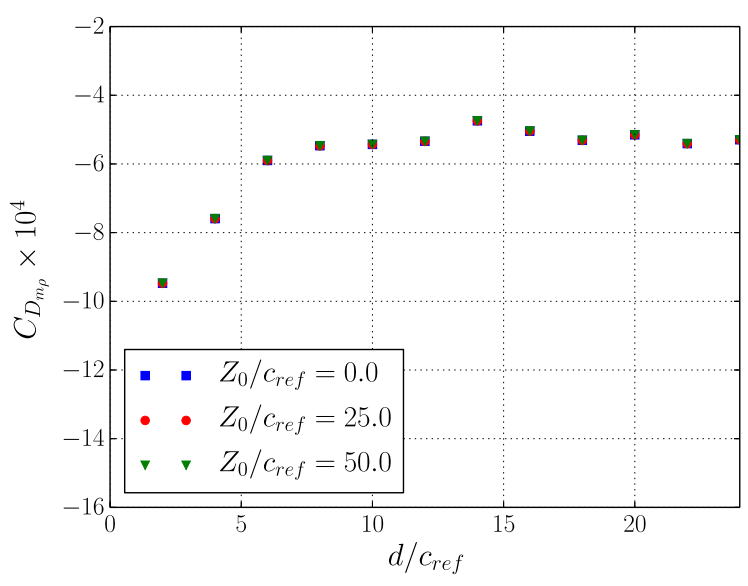

(d) $Z \rightarrow Z+Z_{0}$, new formula

Fig. $13 C_{D_{m_{\rho}}}$ evolutions for the NASA CRM, $M_{\infty}=0.85, R e=5 \times 10^{6}$ and $C_{L}=0.5$ 


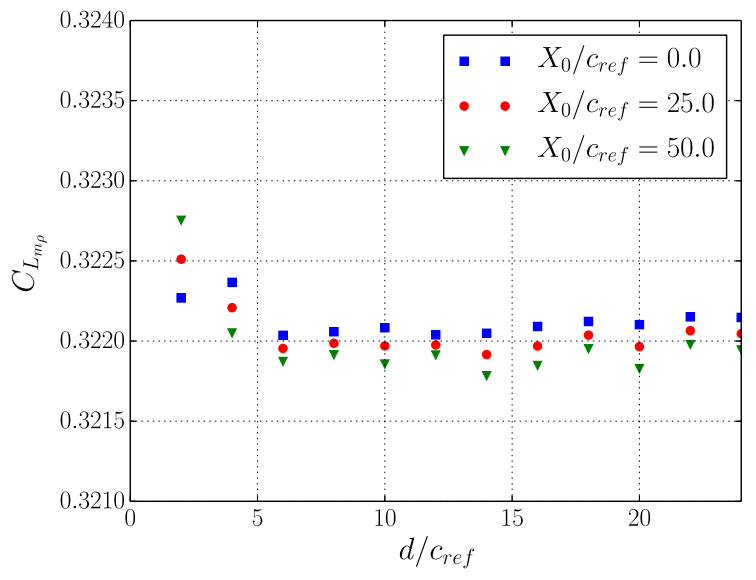

(a) $X \rightarrow X+X_{0}$, old formula

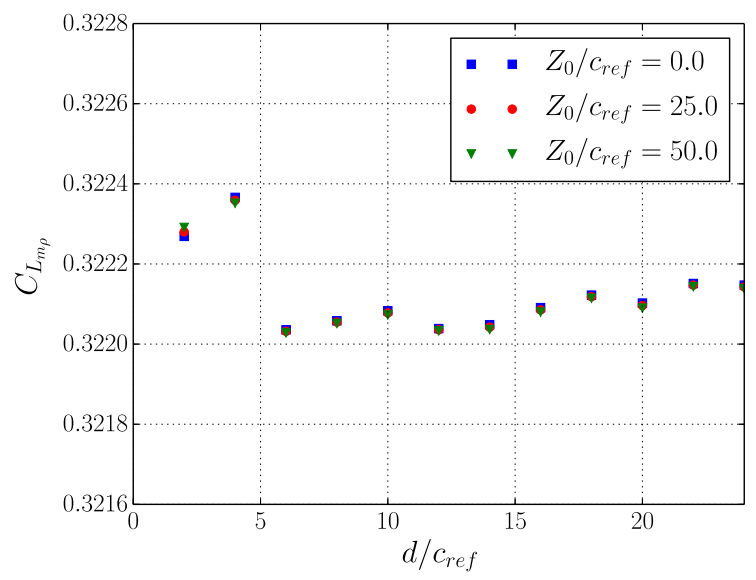

(c) $Z \rightarrow Z+Z_{0}$, old formula

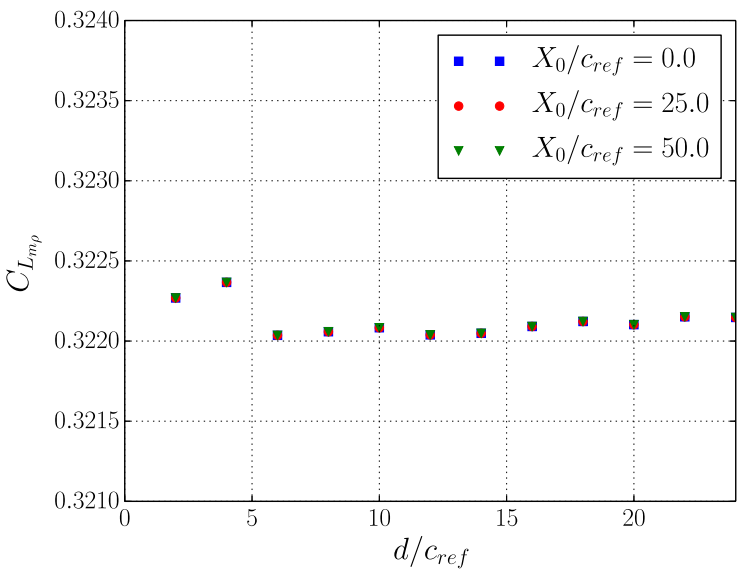

(b) $X \rightarrow X+X_{0}$, new formula

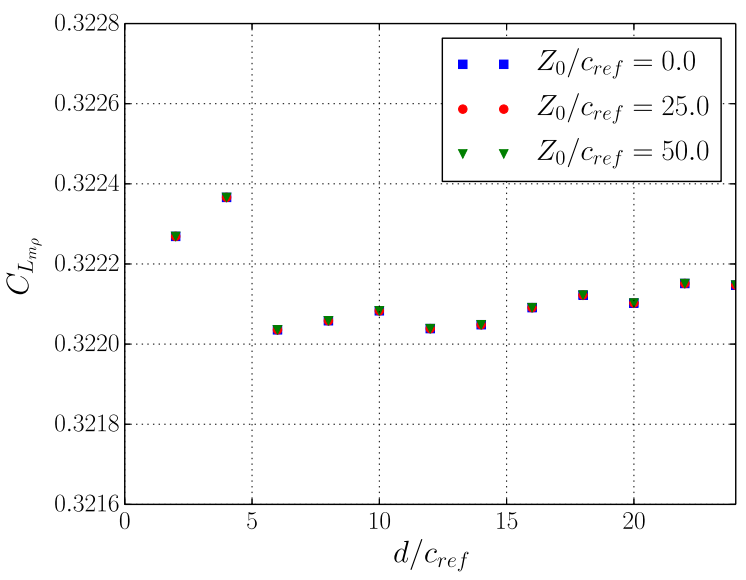

(d) $Z \rightarrow Z+Z_{0}$, new formula

Fig. $14 C_{L_{m_{\rho}}}$ evolutions for the NASA CRM, $M_{\infty}=0.85, R e=5 \times 10^{6}$ and $C_{L}=0.5$

\section{Conclusion}

In the present paper, the focus has been given to the limitations of the Lamb-vector-based aerodynamic force breakdown. In the recent years, this formulation proved to be very promising since it defines the lift and lift-induced drag in compressible viscous flows. However, the sensitivity to the position of the reference point and to the choice of the integration domain is still an open issue which questions the robustness of the method and its potential use.

The present study proposes a way to eliminate the dependence on the reference point by considering the symmetries satisfied by the flow in the far field and extending it to the whole domain such that the additional terms appearing when proceeding to a shift in the reference point vanish. It consists in integrating the part of the physical vector fields that satisfy the symmetries everywhere in the flow. This systematic method may also apply for external boundaries that do 
not necessarily fulfill symmetries since the method presented above can be used to perform a symmetry operation on the surface.

Those symmetries provided a partial theoretical explanation to the fact that the force breakdown progressively becomes domain invariant as the size of the control volume is increased. However, numerical results showed that the increase in the lift-induced drag in the very near wake is mainly caused by the increase of the vortex force contribution whereas the compressibility correction term quickly reaches a constant value. Moreover, it has been shown that the increment in the vortex force contribution fades away because the Lamb vector field progressively satisfies symmetries in the far wake. As a consequence, the symmetries fulfilled by the flow explain why the Lamb-vector-based drag breakdown becomes domain invariant in the mid-field region where the dissipation is weak enough such that the transfer from lift-induced to profile drag is negligible.

The numerical applications were carried out on two-dimensional and three-dimensional cases which allowed the present authors to verify that enforcing the symmetry properties everywhere in the control volume make the formulation independent of the reference point. Therefore, the reference point adopted for the computation of moment transformations can be chosen arbitrarily and the new formulation guarantees more robustness for the Lamb-vector-based aerodynamic force breakdown.

\section{Appendix A: Derivative moment transformations}

Let $f$ be a differentiable vector field, $\Phi$ be a differentiable scalar field, $\Omega$ be a domain bounded by $\partial \Omega$ and $S$ be a surface bounded by $\partial S$. The derivative moment transformations state that in an $n$-dimensional space with $n=2,3$ :

$$
\begin{aligned}
\int_{\Omega} \boldsymbol{f} \mathrm{d} v & =\frac{1}{n-1} \int_{\Omega} \boldsymbol{r} \times(\nabla \times \boldsymbol{f}) \mathrm{d} v-\frac{1}{n-1} \oint_{\partial \Omega} \boldsymbol{r} \times(\boldsymbol{n} \times \boldsymbol{f}) \mathrm{d} S \\
\int_{S} \Phi \boldsymbol{n} \mathrm{d} S & =-\frac{1}{n-1} \int_{S} \boldsymbol{r} \times(\boldsymbol{n} \times \nabla \Phi) \mathrm{d} S+\frac{1}{n-1} \oint_{\partial S} \Phi \boldsymbol{r} \times \mathrm{d} \boldsymbol{r}
\end{aligned}
$$

Those identities are $\boldsymbol{r}_{0}$-independent: a shift $\boldsymbol{r} \rightarrow \boldsymbol{r}+\boldsymbol{r}_{0}$ gives birth to two additional terms that cancel each other according to Gauss theorem. However, the right-hand side integrals depend on the choice of $\boldsymbol{r}_{0}$.

\section{Appendix B: Far wake symmetry properties of the velocity vector derivatives}

Let $\mathcal{F}$ be a differentiable tensor and $\left(x_{1}, \ldots, x_{i}, \ldots, x_{n}\right)$ be a cartesian coordinate system. Then, the derivative of $\mathcal{F}$ with respect to $x_{i}$ writes:

$$
\frac{\partial \mathcal{F}}{\partial x_{i}}\left(x_{1}, \ldots, x_{i}, \ldots, x_{n}\right)=\lim _{\epsilon \rightarrow 0} \frac{\mathcal{F}\left(x_{1}, \ldots, x_{i}+\epsilon, \ldots x_{n}\right)-\mathcal{F}\left(x_{1}, \ldots, x_{i}, \ldots x_{n}\right)}{\epsilon}
$$


Therefore, the derivative of $\mathcal{F}$ with respect to $x_{i}$ assessed in $\left(x_{1}, \ldots,-x_{i}, \ldots, x_{n}\right)$ writes:

$$
\begin{aligned}
\frac{\partial \mathcal{F}}{\partial x_{i}}\left(x_{1}, \ldots,-x_{i}, \ldots, x_{n}\right) & =\lim _{\epsilon \rightarrow 0} \frac{\mathcal{F}\left(x_{1}, \ldots,-x_{i}+\epsilon, \ldots x_{n}\right)-\mathcal{F}\left(x_{1}, \ldots,-x_{i}, \ldots x_{n}\right)}{\epsilon} \\
& =\lim _{\epsilon \rightarrow 0} \frac{\mathcal{F}\left(x_{1}, \ldots,-\left(x_{i}-\epsilon\right), \ldots x_{n}\right)-\mathcal{F}\left(x_{1}, \ldots,-x_{i}, \ldots x_{n}\right)}{\epsilon}
\end{aligned}
$$

Starting from Eqs.28a and 28a and using Eqs. B.1] and [B.2], it is possible to derive the symmetry properties satisfied by the velocity vector derivatives:

$$
\left\{\begin{array} { l } 
{ \frac { \partial \boldsymbol { u } } { \partial x } ( x , - y , z ) = \boldsymbol { S } _ { y } \cdot \frac { \partial \boldsymbol { u } } { \partial x } ( x , y , z ) } \\
{ \frac { \partial \boldsymbol { u } } { \partial y } ( x , - y , z ) = - \boldsymbol { S } _ { y } \cdot \frac { \partial \boldsymbol { u } } { \partial y } ( x , y , z ) } \\
{ \frac { \partial \boldsymbol { u } } { \partial z } ( x , - y , z ) = \boldsymbol { S } _ { y } \cdot \frac { \partial \boldsymbol { u } } { \partial z } ( x , y , z ) }
\end{array} \quad \text { and } \quad \left\{\begin{array}{l}
\frac{\partial \boldsymbol{u}}{\partial x}(x, y,-z)=\boldsymbol{S}_{y} \cdot \frac{\partial \boldsymbol{u}}{\partial x}(x, y, z) \\
\frac{\partial \boldsymbol{u}}{\partial y}(x, y,-z)=\boldsymbol{S}_{y} \cdot \frac{\partial \boldsymbol{u}}{\partial y}(x, y, z) \\
\frac{\partial \boldsymbol{u}}{\partial z}(x, y,-z)=-\boldsymbol{S}_{y} \cdot \frac{\partial \boldsymbol{u}}{\partial z}(x, y, z)
\end{array}\right.\right.
$$

\section{Appendix C: Grid convergence studies}

This appendix presents grid convergence studies on the meshes used in this paper. The effect of grid sizing is sought on the total force computed by the Lamb-vector-based formula and a comparison is made with near-field results. The finest mesh was used in the case of the OAT15A airfoil while, in the case of the NASA CRM, the L4' grid was used since it is a well refined grid.

All the OAT15A airfoil grids have a $y+\approx 0.5$ for which the far field lies 50,000 chords away from the surface. For the NASA CRM, the $\mathrm{L} 2^{\prime}$ has a $y+=1.33$, the $\mathrm{L} 3^{\prime}$ has a $y+=1$, the $\mathrm{L} 4^{\prime}$ has a $y+=0.67$ and the $\mathrm{L}^{\prime}$ has a $y+=0.5$ [45].

\begin{tabular}{lccc}
\hline \hline Nb of points & 41,026 & 115,530 & 312,082 \\
\hline \multicolumn{4}{c}{$C_{D} \times 10^{4}$} \\
\hline$C_{D_{\rho l}}$ & 114.42 & 115.93 & 116.45 \\
$C_{D_{m_{\rho}}}$ & -115.40 & -115.94 & -116.18 \\
$C_{D_{S_{e}}}$ & 124.02 & 120.85 & 119.79 \\
Total & 123.04 & 120.84 & 120.07 \\
Near-field & 119.68 & 117.79 & 117.55 \\
\hline \multicolumn{4}{c}{$C_{L}$} \\
\hline$C_{L_{\rho l}}$ & 0.35049 & 0.35342 & 0.35432 \\
$C_{L_{m}}$ & 0.36300 & 0.36806 & 0.36941 \\
$C_{L_{S}}$ & -0.00049 & -0.00031 & -0.00024 \\
Total & 0.71300 & 0.72117 & 0.72349 \\
Near-field & 0.71330 & 0.72137 & 0.72366 \\
\hline \hline
\end{tabular}

Table 1 Effect of the OAT15A airfoil grid sizing on the force prediction and breakdown, $d / c=10$ 


\begin{tabular}{lcccc}
\hline \hline Grid level & $\mathrm{L}^{\prime}$ & $\mathrm{L}^{\prime}$ & $\mathrm{L}^{\prime}$ & $\mathrm{L}^{\prime}$ \\
\hline \multicolumn{5}{c}{$C_{D} \times 10^{4}$} \\
\hline$C_{D_{\rho l}}$ & 85.10 & 86.74 & 88.61 & 89.24 \\
$C_{D_{m_{\rho}}}$ & -2.67 & -3.21 & -4.53 & -5.18 \\
$C_{D_{S_{e}}}$ & 185.43 & 179.58 & 174.72 & 173.34 \\
Total & 267.86 & 263.12 & 258.80 & 257.41 \\
Near-field & 258.38 & 256.34 & 254.70 & 254.35 \\
\hline \multicolumn{5}{c}{$C_{L}$} \\
\hline$C_{L_{\rho l}}$ & 0.17884 & 0.17911 & 0.17954 & 0.17940 \\
$C_{L_{m}}$ & 0.31202 & 0.31434 & 0.31695 & 0.31855 \\
$C_{L_{S}}$ & -0.00270 & -0.00234 & -0.00233 & -0.00212 \\
Total & 0.48816 & 0.49111 & 0.49416 & 0.49583 \\
Near-field & 0.49967 & 0.49990 & 0.49946 & 0.49963 \\
\hline \hline
\end{tabular}

Table 2 Effect of the NASA CRM grid sizing on the force prediction and breakdown, $d / c_{r e f}=2$

\section{Acknowledgments}

Part of this research was supported by a DGA scholarship. The authors would also like to thank Ilias Petropoulos from

ONERA, Meudon, France for providing the CFD solutions around the NASA CRM.

\section{References}

[1] Joukowski, N., “On annexed vortices,” Proc. Phys. Section of the Natural Science Society, Vol. 13, No. 2, 1906, pp. $12-25$.

[2] Ackroyd, J. A., Axcell, B. P., and Ruban, A., Early developments of modern aerodynamics, American Institute of Aeronautics and Astronautics / Butterworth-Heinemann, 2001.

[3] Wu, J. Z., Ma, H. Y., and Zhou, M. D., Vortical flows, Vol. 28, Springer, 2015. doi:10.1007/978-3-662-47061-9.

[4] Prandtl, L., “Theory of lifting surfaces," Tech. Rep. NACA TN10, National Advisory Committee for Aeronautics, 1919.

[5] Filon, L. N. G., "The forces on a cylinder in a stream of viscous fluid," Proceedings of the Royal Society of London. Series A, Containing Papers of a Mathematical and Physical Character, Vol. 113, No. 763, 1926, pp. 7-27. doi:10.1098/rspa.1926.0136.

[6] Liu, L. Q., Zhu, J. Y., and Wu, J. Z., "Lift and drag in two-dimensional steady viscous and compressible flow," Journal of Fluid Mechanics, Vol. 784, 2015, pp. 304-341. doi:10.1017/jfm.2015.584.

[7] Liu, L. Q., Wu, J. Z., Su, W. D., and Kang, L. L., "Lift and drag in three-dimensional steady viscous and compressible flow," Physics of Fluids, Vol. 29, No. 11, 2017, p. 116105. doi:10.1063/1.4989747.

[8] Schmitz, S., "Finite Domain Viscous Correction to the Kutta-Joukowski Theorem in Incompressible Flow," AIAA Journal, Vol. 52, No. 9, 2014, pp. 2079-2083. doi:10.2514/1.J053114. 
[9] Méheut, M., and Bailly, D., "Drag-breakdown methods from wake measurements," AIAA journal, Vol. 46, No. 4, 2008, pp. 847-862. doi:10.2514/1.29051.

[10] Maskell, E., "Progress Towards a Method for the Measurement of the Components of the Drag of a Wing of Finite Span," Tech. Rep. 72232, Procurement Executive, Ministry of Defence, Royal Aircraft Establishment, 1972.

[11] Wu, J. Z., Liu, L. Q., and Liu, T. S., "Fundamental theories of aerodynamic force in viscous and compressible complex flows," Progress in Aerospace Sciences, 2018. doi:10.1016/j.paerosci.2018.04.002.

[12] Cummings, R. M., Giles, M. B., and Shrinivas, G., "Analysis of the elements of drag in three-dimensional viscous and inviscid flows," 14th Applied Aerodynamics Conference, 1996, p. 2482. doi:10.2514/6.1996-2482.

[13] Giles, M. B., and Cummings, R. M., "Wake integration for three-dimensional flowfield computations: theoretical development," Journal of aircraft, Vol. 36, No. 2, 1999, pp. 357-365. doi:10.2514/2.2465.

[14] Van Dam, C. P., "Recent experience with different methods of drag prediction," Progress in Aerospace Sciences, Vol. 35, No. 8, 1999, pp. 751-798. doi:10.1016/S0376-0421(99)00009-3.

[15] Kroo, I., "Drag due to lift: concepts for prediction and reduction," Annual review of fluid mechanics, Vol. 33, No. 1, 2001, pp. 587-617. doi:10.1146/annurev.fluid.33.1.587.

[16] Kusunose, K., and Crowder, J. P., "Extension of wake-survey analysis method to cover compressible flows," Journal of aircraft, Vol. 39, No. 6, 2002, pp. 954-963. doi:10.2514/2.3048.

[17] Chao, D., and Van Dam, C., "Wing drag prediction and decomposition," Journal of Aircraft, Vol. 43, No. 1, 2006, pp. 82-90. doi:10.2514/1.12311.

[18] Ueno, M., Yamamoto, K., Tanaka, K., Murayama, M., and Tognaccini, R., "Far-field drag analysis of NASA Common Research Model simulation,” Journal of Aircraft, Vol. 50, No. 2, 2013, pp. 388-397. doi:10.2514/1.C031766.

[19] Betz, A., "A method for the direct determination of wing-section drag," Tech. Rep. NACA TM37, National Advisory Committee for Aeronautics, 1925.

[20] Taylor, G., "Note on the connection between the lift on an aërofoil in a wind and the circulation round it," Phil. Trans. R. Soc. London A, Vol. 225, 1926, pp. 238-246.

[21] Jones, B. M., The measurement of profile drag by the pitot-traverse method, HM Stationery Office, 1936.

[22] Oswatitsch, K., Gas dynamics, Vol. 1, Academic Press, 1956.

[23] Van Der Vooren, J., and Slooff, J., "CFD-based drag prediction: state-of-the-art, theory, prospects," National Aerospace Lab., NLR, TP 90247 U, 1992.

[24] Paparone, L., and Tognaccini, R., "Computational Fluid Dynamics-Based Drag Prediction and Decomposition,” AIAA Journal, Vol. 41, No. 9, 2003, pp. 1647-1657. doi:10.2514/2.7300. 
[25] Destarac, D., "Far-field/near-field drag balance and applications of drag extraction in CFD," VKI Lecture Series, Vol. 2, 2003, pp. 3-7.

[26] Destarac, D., and Van Der Vooren, J., "Drag/thrust analysis of jet-propelled transonic transport aircraft; definition of physical drag components," Aerospace science and technology, Vol. 8, No. 6, 2004, pp. 545-556. doi:10.1016/j.ast.2004.03.004.

[27] Fan, Y., and Li, W., "Review of far-field drag decomposition methods for aircraft design,” Journal of Aircraft, Vol. 56, No. 1, 2019, pp. 11-21. doi:10.2514/1.C034781.

[28] Spalart, P. R., "On the far wake and induced drag of aircraft," Journal of Fluid Mechanics, Vol. 603, 2008 , pp. 413-430. doi:10.1017/S0022112008001146.

[29] Schmitz, S., and Coder, J. G., "Inviscid circulatory-pressure field derived from the incompressible Navier-Stokes equations," AIAA Journal, Vol. 53, No. 1, 2014, pp. 33-41. doi:10.2514/1.J053140.

[30] Schmitz, S., "Drag Decomposition Using Partial-Pressure Fields in the Compressible Navier-Stokes Equations," AIAA Journal, Vol. 57, No. 5, 2018, pp. 2030-2038. doi:10.2514/1.J057701.

[31] Saffman, P. G., Vortex dynamics, Cambridge university press, 1992.

[32] Wu, J. Z., Ma, H. Y., and Zhou, M. D., Vorticity and vortex dynamics, Springer Science \& Business Media, 2007.

[33] Wu, J. Z., Lu, X. Y., and Zhuang, L. X., "Integral force acting on a body due to local flow structures," Journal of Fluid Mechanics, Vol. 576, 2007, pp. 265-286. doi:10.1017/S0022112006004551.

[34] Liu, L. Q., Wu, J. Z., Shi, Y. P., and Zhu, J. Y., "A dynamic counterpart of Lamb vector in viscous compressible aerodynamics," Fluid Dynamics Research, Vol. 46, No. 6, 2014, p. 061417. doi:10.1088/0169-5983/46/6/061417.

[35] Mele, B., and Tognaccini, R., “Aerodynamic force by Lamb vector integrals in compressible flow,” Physics of Fluids, Vol. 26, No. 5, 2014, p. 056104. doi:10.1063/1.4875015.

[36] Marongiu, C., Tognaccini, R., and Ueno, M., "Lift and lift-induced drag computation by Lamb vector integration," AIAA Journal, Vol. 51, No. 6, 2013, pp. 1420-1430. doi:10.2514/1.J052104.

[37] Yang, Y. T., Zhang, R. K., An, Y. R., and Wu, J. Z., "Steady vortex force theory and slender-wing flow diagnosis," Acta Mechanica Sinica, Vol. 23, No. 6, 2007, pp. 609-619. doi:10.1007/s10409-007-0107-0.

[38] Mele, B., Ostieri, M., and Tognaccini, R., "Aircraft lift and drag decomposition in transonic flows," Journal of Aircraft, Vol. 54, No. 5, 2017, pp. 1933-1944. doi:10.2514/1.C034288.

[39] Ostieri, M., and Tognaccini, R., “On a recently proposed vorticity-based definition of wave drag," Journal of Aircraft, Vol. 55, No. 6, 2018, pp. 2521-2523. doi:10.2514/1.C034820.

[40] Zou, S. F., Wu, J. Z., Gao, A. K., Liu, L. Q., Kang, L. L., and Shi, Y. P., “On the concept and theory of induced drag for viscous and incompressible steady flow," Physics of Fluids, Vol. 31, No. 6, 2019, p. 065106. doi:10.1063/1.5090165. 
[41] Kang, L. L., Russo, L., Tognaccini, R., Wu, J. Z., and Su, W. D., "Aerodynamic Force Breakdown in Reversible and Irreversible Components by Vortex Force Theory," AIAA Journal, Vol. 57, No. 11, 2019, pp. 4623-4638. doi:10.2514/1.J058379.

[42] Mele, B., Ostieri, M., and Tognaccini, R., "Vorticity based breakdown of the aerodynamic force in three-dimensional compressible flows," AIAA Journal, Vol. 54, No. 4, 2016, pp. 1198-1208. doi:10.2514/1.J054363.

[43] Cambier, L., Heib, S., and Plot, S., "The Onera elsA CFD software: input from research and feedback from industry," Mechanics $\mathcal{E}$ Industry, Vol. 14, No. 3, 2013, pp. 159-174. doi:10.1051/meca/2013056.

[44] Fournis, C., Bailly, D., and Tognaccini, R., "A reference point invariant Lamb vector based aerodynamic force breakdown in steady compressible flows," AIAA Scitech 2020 Forum, 2020. doi:10.2514/6.2020-1996.

[45] Hue, D., "Fifth drag prediction workshop: ONERA investigations with experimental wing twist and laminarity," Journal of Aircraft, Vol. 51, No. 4, 2014, pp. 1311-1322. doi:10.2514/1.C032438.

[46] Vassberg, J., "A Unified Baseline Grid about the Common Research Model Wing/Body for the Fifth AIAA CFD Drag Prediction Workshop," 29th AIAA Applied Aerodynamics Conference, 2011, p. 3508. doi:10.2514/6.2011-3508.

[47] Devenport, W. J., Rife, M. C., Liapis, S. I., and Follin, G. J., “The structure and development of a wing-tip vortex,” Journal of Fluid Mechanics, Vol. 312, 1996, pp. 67-106. doi:10.1017/S0022112096001929.

[48] Batchelor, G., "Axial flow in trailing line vortices," Journal of Fluid Mechanics, Vol. 20, No. 04, 1964, pp. 645-658. doi: $10.1017 / \mathrm{S} 0022112064001446$.

[49] Fabre, D., "Instabilité et instationnarités dans les tourbillons: Application aux sillages d'avions," Ph.D. thesis, Université Paris $6,2002$.

[50] Misaka, T., Holzäpfel, F., and Gerz, T., "Large-eddy simulation of aircraft wake evolution from roll-up until vortex decay," AIAA Journal, Vol. 53, No. 9, 2015, pp. 2646-2670. doi:10.2514/1.J053671.

[51] Liu, L. Q., Kang, L. L., and Wu, J. Z., “Zonal structure of unbounded external-flow and aerodynamics,” Fluid Dynamics Research, Vol. 49, No. 4, 2017, p. 045508. doi:10.1088/1873-7005/aa79d0.

[52] Liu, L. Q., Unified Theoretical Foundations of Lift and Drag in Viscous and Compressible External Flows, Springer, 2017. doi:10.1007/978-981-10-6223-0.

[53] Sipp, D., Jacquin, L., and Cossu, C., "Self-adaptation and viscous selection in concentrated two-dimensional vortex dipoles," Physics of Fluids, Vol. 12, No. 2, 2000, pp. 245-248. doi:10.1063/1.870325.

[54] Schmitt, V., and Destarac, D., "Recent progress in drag prediction and reduction for civil transport aircraft at ONERA," 36th AIAA Aerospace Sciences Meeting and Exhibit, 1998, p. 137. doi:10.2514/6.1998-137. 